\title{
On a Time Domain Boundary Integral Equation Formulation for Acoustic Scattering by Rigid Bodies in Uniform Mean Flow
}

\author{
Fang Q.Hu \\ Old Dominion University, fhu@odu.edu \\ Michelle E. Pizzo \\ Old Dominion University \\ Douglas M. Nark
}

Follow this and additional works at: https://digitalcommons.odu.edu/mathstat_fac_pubs
Part of the Acoustics, Dynamics, and Controls Commons, Applied Mathematics Commons, and the Mathematics Commons

\section{Repository Citation}

Hu, Fang Q.; Pizzo, Michelle E.; and Nark, Douglas M., "On a Time Domain Boundary Integral Equation Formulation for Acoustic Scattering by Rigid Bodies in Uniform Mean Flow" (2017). Mathematics \& Statistics Faculty Publications. 64.

https://digitalcommons.odu.edu/mathstat_fac_pubs/64

\section{Original Publication Citation}

Hu, F. Q. Pizzo, M. E., \& Nark, D. M. (2017). On a time domain boundary integral equation formulation for acoustic scattering by rigid bodies in uniform mean flow. Journal of the Acoustical Society of America, 142(6), 3624-3636. doi:10.1121/1.5017734 


\title{
On a time domain boundary integral equation formulation for acoustic scattering by rigid bodies in uniform mean flow
}

\author{
Fang Q. Hu ${ }^{a)}$ and Michelle E. Pizzo \\ Department of Mathematics and Statistics, Old Dominion University, Norfolk, Virginia 23529, USA \\ Douglas M. Nark \\ Structural Acoustics Branch, NASA Langley Research Center, Hampton, Virginia 23681, USA
}

(Received 24 June 2017; revised 12 October 2017; accepted 26 November 2017; published online 18 December 2017)

\begin{abstract}
It has been well-known that under the assumption of a uniform mean flow, the acoustic wave propagation equation can be formulated as a boundary integral equation. However, the constant mean flow assumption, while convenient for formulating the integral equation, does not satisfy the solid wall boundary condition wherever the body surface is not aligned with the assumed uniform flow. A customary boundary condition for rigid surfaces is that the normal acoustic velocity be zero. In this paper, a careful study of the acoustic energy conservation equation is presented that shows such a boundary condition would in fact lead to source or sink points on solid surfaces. An alternative solid wall boundary condition, termed zero energy flux boundary condition, is proposed that conserves the acoustic energy and a time domain boundary integral equation is derived. Furthermore, stabilization of the integral equation by Burton-Miller type reformulation is presented. The stability is studied theoretically as well as numerically by an eigenvalue analysis. Numerical solutions are also presented that demonstrate the stability of the current formulation.
\end{abstract} (C) 2017 Acoustical Society of America. https://doi.org/10.1121/1.5017734

[NAG]

Pages: $3624-3636$

\section{INTRODUCTION}

Numerical solution of sound scattering by an acoustically large body remains a significant challenge due to its high demand on computational resources that are required to resolve the acoustic waves of short wavelengths. It is wellknown that under the assumption of a constant mean flow, the acoustic wave propagation is governed by the convective wave equation that, in turn, can be converted into a boundary integral equation. The boundary integral equation approach has the advantage of reducing the spatial dimensions of the problem by one, making it an attractive computational method for calculating sound scattering and shielding at mid to high frequencies. In this paper, we consider the problem of acoustic scattering by rigid bodies in the presence of a uniform flow using the boundary integral equation approach. The present approach is based on the time domain boundary integral equation. The time domain approach has some distinct advantages over a frequency domain approach. Most notably, scattering solutions at all frequencies are obtained within one single computation. In addition, broadband noise sources and time dependent transient signals can be simulated and studied. The time domain approach also couples naturally with nonlinear computations where many frequencies are generated.

Previously, scattering of sound waves by rigid bodies with flow has been studied, in both the frequency domain and the time domain. In Ref. 1, acoustic radiation in a moving flow was formulated as a boundary integral equation in

\footnotetext{
${ }^{a)}$ Electronic mail: fhu@odu.edu
}

the frequency domain. The nonuniqueness of the exterior problem was dealt with by applying the Burton-Miller reformulation procedure. ${ }^{2}$ In the time domain, a boundary integral equation approach for scattering by moving surfaces was first formulated and studied in Ref. 3. More recent studies of the time domain approach in the presence of a mean flow can be found in Refs. 4-6.

A major difference between the current approach and those taken previously is in the treatment of the boundary condition at solid surfaces in the presence of flow. While the linear acoustic problem as a perturbation over the mean flow can be considered separately from the mean flow, an implicit condition is that the mean flow itself satisfies the solid wall boundary condition. The assumption of a constant mean flow is an approximation to the actual mean flow and this assumption is made such that the formulation of a boundary integral equation becomes possible. While this facilitates the conversion of the partial differential equation to the boundary integral equation, the simplified mean flow itself obviously cannot satisfy the physical boundary condition at solid boundaries wherever the surface is not aligned with the assumed constant mean flow. As pointed out in Ref. 3, the boundary integral equation derived based on such an assumption would be formally valid when $M_{n} \ll 1$, where $M_{n}$ is the Mach number of mean flow normal to the body surface. In this paper, we take a closer look at the boundary condition to be used for scattering of acoustic waves at solid surfaces where $M_{n}$ is nonzero. In all the previous studies, a boundary condition of normal acoustic velocity being zero has been applied everywhere including the surfaces where $M_{n} \neq 0$. However, an analysis of the acoustic energy equation will show that the usual boundary condition would lead 
to nonzero energy flux at surfaces where $M_{n} \neq 0$, which could potentially lead to nonconservation of the acoustic energy. A new formulation is derived based on this acoustic energy consideration, and an alternative boundary condition is proposed by the requirement that energy flux be zero at solid surfaces. From a physical point of view, the null acoustic energy flux condition should be equivalent to, or a direct consequence of, the condition that the normal acoustic velocity becomes zero on rigid surfaces. The fact that the two now differ in the formulation of the boundary integral equation for scattering with flow is due to the inconsistency on the part of the underlying mean flow itself when the constant flow simplification is made. Naturally, as mentioned earlier, boundary integral equation approaches with a constant mean flow would be applicable only to problems where such a simplification is acceptable or justified, such as in scattering with flow over slender bodies. From a computational point of view, however, due to the structure of the integral equation, the new formulation also becomes much simpler than those found in the literature for scattering with flow, which is of great benefit for computation.

In addition to the modification of boundary condition at solid surfaces, a Burton-Miller type reformulation of the integral equation consistent with the new boundary condition is also presented. It is well-known that the direct solution of boundary integral equation for exterior scattering problems is prone to numerical instabilities. ${ }^{1,2,4,7-12}$ In the time domain, the instability is also more easily excited because all frequencies within the numerical resolution are present in the computation. There are generally two approaches for dealing with this instability. One is the Burton-Miller reformulation which has been widely used for frequency domain exterior scattering problems. Recently, it has been shown that Burton-Miller reformulation is effective for time domain as well. ${ }^{7,8,11}$ Another method for the removal of the instability is the CHIEF method. ${ }^{12,13}$ In the present study, we apply the Burton-Miller technique for the elimination of instabilities.

The rest of the paper is organized as follows. In Sec. II, an integral relation for acoustic wave propagation is derived for a constant mean flow in a general direction. Then, the time domain boundary integral equation for scattering by rigid bodies is derived in Sec. III. In Sec. IV, a Burton-Miller type reformulation of time domain boundary integral equation is presented and a discussion on the stability of the new formulation is given in Sec. V. Numerical methods for the time domain boundary integral equation are discussed in Sec. VI. Stability of the current formulation is demonstrated in Sec. VII by analyzing the eigenvalues of the discretized system. An example of scattering by a convex parabolic wing in the presence of a mean flow is presented in Sec. VIII. Section IX contains the conclusions.

\section{INTEGRAL REPRESENTATION OF ACOUSTIC WAVES IN THE PRESENCE OF A UNIFORM MEAN FLOW}

The current problem is considered in the context of solving the wave equation in a moving medium exterior of certain specified surface $S$, such as the scattering of sound field by an object as shown in Fig. 1. Acoustic waves are assumed to be disturbances of small amplitudes. Linear acoustic problems are frequently formulated using a velocity potential function $\phi(\boldsymbol{r}, t)$ where the acoustic velocity $\boldsymbol{u}$ and pressure $p$ are related to $\phi$ as follows:

$$
\boldsymbol{u}=\nabla \phi, \quad p=-\rho_{0}\left(\frac{\partial \phi}{\partial t}+\boldsymbol{U} \cdot \nabla \phi\right),
$$

where $\rho_{0}$ is the mean density. With a constant mean flow $\boldsymbol{U}$, the acoustic disturbances are governed by the convective wave equation. ${ }^{14}$ In the present study, we consider the solution of the following equation for the velocity potential:

$$
\left(\frac{\partial}{\partial t}+\boldsymbol{U} \cdot \nabla\right)^{2} \phi-c^{2} \nabla^{2} \phi=q(\boldsymbol{r}, t),
$$

with homogeneous initial conditions

$$
\phi(\boldsymbol{r}, 0)=\frac{\partial \phi}{\partial t}(\boldsymbol{r}, 0)=0, t=0 .
$$

In the above, $c$ is the speed of sound, $\boldsymbol{U}$ is the constant mean velocity, and $q(\boldsymbol{r}, t)$ represents the known acoustic sources. Furthermore, in addition to the radiation condition at the far field, Eqs. (2) and (3) are to be supplemented with boundary conditions on the scattering surface $S$. The suitable boundary conditions to be applied on solid surfaces will be discussed in Sec. III. It is well-known that the convective wave Eq. (2) and the initial condition (3), as well as the boundary conditions, can be reformulated into an integral equation. In the literature, integral representation of sound waves in a moving flow is often derived by making use of generalized functions in a setting of moving bodies in an otherwise undisturbed medium. ${ }^{15-21}$ Here, we present a derivation using a free-space Green's function $\tilde{G}\left(\boldsymbol{r}, t ; \boldsymbol{r}^{\prime}, t^{\prime}\right)$ that, for convenience of discussion, is defined as follows:

$$
\left(\frac{\partial}{\partial t}+\boldsymbol{U} \cdot \nabla\right)^{2} \tilde{G}-c^{2} \nabla^{2} \tilde{G}=\delta\left(\boldsymbol{r}-\boldsymbol{r}^{\prime}\right) \delta\left(t-t^{\prime}\right)
$$

with initial conditions

$$
\tilde{G}\left(\boldsymbol{r}, t ; \boldsymbol{r}^{\prime}, t^{\prime}\right)=\frac{\partial \tilde{G}}{\partial t}\left(\boldsymbol{r}, t ; \boldsymbol{r}^{\prime}, t^{\prime}\right)=0, \quad t>t^{\prime},
$$
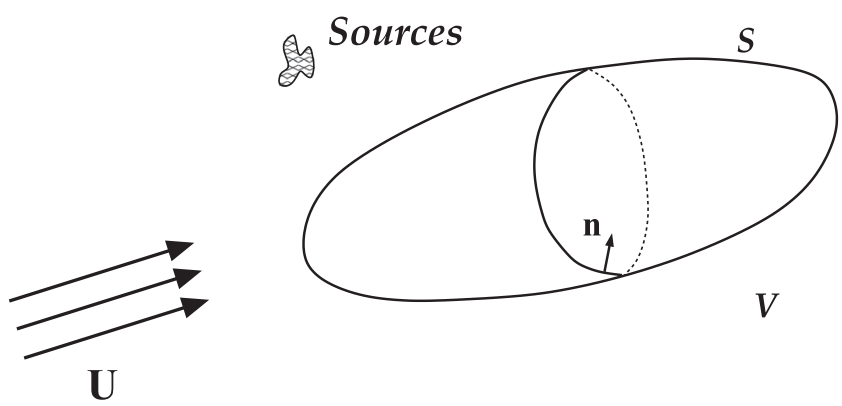

FIG. 1. A schematic showing the scattering body and mean flow. Scattering surface is denoted by $S$ and the solution domain exterior of $S$ is denoted by $V$. The surface normal vector $\boldsymbol{n}$ is taken to be outward from $V$ and thus inward toward the interior of the body. 
where $\boldsymbol{r}^{\prime}$ and $t^{\prime}$ indicate, respectively, the source point and initial time and $\boldsymbol{r}$ and $t$ are the space-time variables of the Green's function.

Note that the time domain Green's function $\tilde{G}\left(\boldsymbol{r}, t ; \boldsymbol{r}^{\prime}, t^{\prime}\right)$ defined above is nonzero for $t \in\left(-\infty, t^{\prime}\right]$. The solution to Eqs. (4) and (5) is well-known (see, e.g., Refs. 14, 17, and 23 ) and, for a mean flow of a general direction, can be written as

$$
\tilde{G}\left(\boldsymbol{r}, t ; \boldsymbol{r}^{\prime}, t^{\prime}\right)=\frac{G_{0}}{4 \pi c^{2}} \delta\left(t^{\prime}-t+\boldsymbol{\beta} \cdot\left(\boldsymbol{r}^{\prime}-\boldsymbol{r}\right)-\frac{\bar{R}}{c \alpha^{2}}\right)
$$

where

$$
\begin{aligned}
& G_{0}=\frac{1}{\bar{R}\left(\boldsymbol{r}, \boldsymbol{r}^{\prime}\right)}, \text { and } \\
& \bar{R}\left(\boldsymbol{r}, \boldsymbol{r}^{\prime}\right)=\sqrt{\left[\boldsymbol{M} \cdot\left(\boldsymbol{r}-\boldsymbol{r}^{\prime}\right)\right]^{2}+\alpha^{2}\left|\boldsymbol{r}-\boldsymbol{r}^{\prime}\right|^{2}},
\end{aligned}
$$

in which

$$
\begin{aligned}
\boldsymbol{M} & =\frac{\boldsymbol{U}}{c}, \quad \alpha=\sqrt{1-M^{2}}, \quad \boldsymbol{\beta}=\frac{\boldsymbol{U}}{c^{2}-U^{2}}=\frac{\boldsymbol{U}}{c^{2} \alpha^{2}}=\frac{\boldsymbol{M}}{c \alpha^{2}}, \\
U & =|\boldsymbol{U}|, \quad M=|\boldsymbol{M}| .
\end{aligned}
$$

By an operation of $\tilde{G} \times(2)-\phi \times(4)$ and by integrating over the volume $V$ exterior of scattering surface $S$ for space and an interval $\left[0^{-}, t^{\prime+}\right]$ for time $t$, it is straightforward to show that we will get

$$
\begin{aligned}
& \int_{0^{-}}^{t^{++}} \int_{V}\left\{\frac{\partial}{\partial t}\left[\tilde{G}\left(\frac{\partial \phi}{\partial t}+\boldsymbol{U} \cdot \nabla \phi\right)-\phi\left(\frac{\partial \tilde{G}}{\partial t}+\boldsymbol{U} \cdot \nabla \tilde{G}\right)\right]\right. \\
& \quad+\nabla \cdot\left[\left(\tilde{G}\left(\frac{\partial \phi}{\partial t}+\boldsymbol{U} \cdot \nabla \phi\right)-\phi\left(\frac{\partial \tilde{G}}{\partial t}+\boldsymbol{U} \cdot \nabla \tilde{G}\right)\right) \boldsymbol{U}\right] \\
& \left.\quad-c^{2} \nabla \cdot[\tilde{G} \nabla \phi-\phi \nabla \tilde{G}]\right\} d \boldsymbol{r} d t \\
& =\int_{0^{-}}^{t^{++}} \int_{V}\left[\tilde{G} q(\boldsymbol{r}, t)-\phi(\boldsymbol{r}, t) \delta\left(\boldsymbol{r}-\boldsymbol{r}^{\prime}\right) \delta\left(t-t^{\prime}\right)\right] d \boldsymbol{r} d t .
\end{aligned}
$$

Integration of the first term in the above will be zero by initial conditions thus defined for $\phi$ and $\tilde{G}$. Then, upon using the divergence theorem and the condition at infinity, we get an expression for $\phi$ at an arbitrary point $\boldsymbol{r}^{\prime}$ in $V$ and time $t^{\prime}$ as follows:

$$
\begin{aligned}
\phi\left(\boldsymbol{r}^{\prime}, t^{\prime}\right)= & \int_{0^{-}}^{t^{++}} \int_{V} \tilde{G} q(\boldsymbol{r}, t) d \boldsymbol{r} d t \\
& +c^{2} \int_{0^{-}}^{t^{++}} \int_{S}\left(\tilde{G} \frac{\partial \phi}{\partial n}-\phi \frac{\partial \tilde{G}}{\partial n}\right) d \boldsymbol{r}_{s} d t \\
& -c \int_{0^{-}}^{t^{++}} \int_{S}\left[\tilde{G}\left(\frac{\partial \phi}{\partial t}+\boldsymbol{U} \cdot \nabla \phi\right)\right. \\
& \left.-\phi\left(\frac{\partial \tilde{G}}{\partial t}+\boldsymbol{U} \cdot \nabla \tilde{G}\right)\right] M_{n} d \boldsymbol{r}_{s} d t
\end{aligned}
$$

where $\boldsymbol{r}_{S}$ denotes points on surface $S$, and

$$
M_{n}=\boldsymbol{n} \cdot \boldsymbol{M}=\boldsymbol{n} \cdot \boldsymbol{U} / c
$$

is the normal component of the mean velocity Mach number on surface point $\boldsymbol{r}_{s}$. Here, the unit normal vector $\boldsymbol{n}$ is assumed to be outward from the solution domain. For the exterior scattering problem considered in the present study, the normal vector is then the one that is inward to the body as noted in Fig. 1.

For convenience of discussion, we define a modified normal derivative (denoted by an overbar) as

$$
\frac{\partial}{\partial \bar{n}}=\frac{\partial}{\partial n}-M_{n}(\boldsymbol{M} \cdot \nabla)
$$

Then, Eq. (9) can be written as

$$
\begin{aligned}
\phi\left(\boldsymbol{r}^{\prime}, t^{\prime}\right)= & \int_{0}^{t^{\prime+}} \int_{V} \tilde{G} q(\boldsymbol{r}, t) d \boldsymbol{r} d t \\
& +c^{2} \int_{0}^{t^{\prime+}} \int_{S}\left(\tilde{G} \frac{\partial \phi}{\partial \bar{n}}-\phi \frac{\partial \tilde{G}}{\partial \bar{n}}\right) d \boldsymbol{r}_{s} d t \\
& -c \int_{0}^{t^{\prime+}} \int_{S}\left[\tilde{G} \frac{\partial \phi}{\partial t}-\phi \frac{\partial \tilde{G}}{\partial t}\right] M_{n} d \boldsymbol{r}_{s} d t
\end{aligned}
$$

Furthermore, if we introduce a combined normal derivative (denoted by a tilde) as

$$
\frac{\partial}{\partial \tilde{n}}=\frac{\partial}{\partial n}-\frac{M_{n}}{c}\left(\frac{\partial}{\partial t}+\boldsymbol{U} \cdot \nabla\right)=\frac{\partial}{\partial \bar{n}}-\frac{M_{n}}{c} \frac{\partial}{\partial t},
$$

we get another expression:

$$
\begin{aligned}
\phi\left(\boldsymbol{r}^{\prime}, t^{\prime}\right)= & \int_{0^{-}}^{t^{++}} \int_{V} \tilde{G} q(\boldsymbol{r}, t) d \boldsymbol{r} d t \\
& +c^{2} \int_{0^{-}}^{t^{++}} \int_{S}\left(\tilde{G} \frac{\partial \phi}{\partial \tilde{n}}-\phi \frac{\partial \tilde{G}}{\partial \tilde{n}}\right) d \boldsymbol{r}_{S} d t .
\end{aligned}
$$

Equations (9), (11), or (13) is the Kirchhoff integral representation of the acoustic field in the presence of a uniform mean flow. The integral relation can be further expressed as integration of retarded values by utilizing $\tilde{G}$ as given in Eq. (6). In particular, note that we have

$$
\begin{aligned}
\frac{\partial \tilde{G}}{\partial \tilde{n}}= & \frac{1}{4 \pi c^{2}} \frac{\partial G_{0}}{\partial \bar{n}}\left[\delta\left(t^{\prime}-t+\boldsymbol{\beta} \cdot\left(\boldsymbol{r}^{\prime}-\boldsymbol{r}\right)-\frac{\bar{R}}{c \alpha^{2}}\right)\right. \\
& \left.+\frac{\bar{R}}{c \alpha^{2}} \delta^{\prime}\left(t^{\prime}-t+\boldsymbol{\beta} \cdot\left(\boldsymbol{r}^{\prime}-\boldsymbol{r}\right)-\frac{\bar{R}}{c \alpha^{2}}\right)\right]
\end{aligned}
$$

where $G_{0}$ and $\bar{R}$ are those defined in Eq. (7). Then Eq. (13) can be written as

$$
\begin{aligned}
\phi\left(\boldsymbol{r}^{\prime}, t^{\prime}\right)= & \frac{1}{4 \pi c^{2}} \int_{V_{s}} \frac{1}{\bar{R}} q\left(\boldsymbol{r}, t_{R}^{\prime}\right) d \boldsymbol{r}+\frac{1}{4 \pi} \int_{S}\left[G_{0} \frac{\partial \phi}{\partial \tilde{n}}\left(\boldsymbol{r}_{s}, t_{R}^{\prime}\right)\right. \\
& \left.-\frac{\partial G_{0}}{\partial \bar{n}}\left(\phi\left(\boldsymbol{r}_{s}, t_{R}^{\prime}\right)+\frac{\bar{R}}{c \alpha^{2}} \frac{\partial \phi}{\partial t}\left(\boldsymbol{r}_{s}, t_{R}^{\prime}\right)\right)\right] d \boldsymbol{r}_{s}
\end{aligned}
$$

where $V_{s}$ denotes the region of acoustic sources and the retarded time for $t^{\prime}$ is defined as

$$
t_{R}^{\prime}=t^{\prime}+\boldsymbol{\beta} \cdot\left(\boldsymbol{r}^{\prime}-\boldsymbol{r}\right)-\frac{\bar{R}}{c \alpha^{2}} .
$$


The modified normal derivative for $G_{0}$ is found to be the following:

$$
\frac{\partial G_{0}}{\partial \bar{n}}=-\frac{1}{\bar{R}^{2}} \frac{\partial \bar{R}}{\partial \bar{n}}=-\alpha^{2} \frac{\boldsymbol{n} \cdot\left(\boldsymbol{r}-\boldsymbol{r}^{\prime}\right)}{\bar{R}^{3}} .
$$

Equation (15) relates the solution at point $\boldsymbol{r}^{\prime}$ and time $t^{\prime}$ to the direct contribution from source function $q$ and a surface contribution involving the retarded values of $\phi$ and their normal derivatives. As shown in Ref. 4, this form is equivalent to previous such formulations appearing in the literature, e.g., in Refs. 15 and 19, where the relationship had been derived under the assumption of a mean flow that is aligned with the $x$ axis.

When both $\phi\left(\boldsymbol{r}_{s}, t\right)$ and $(\partial \phi / \partial \tilde{n})\left(\boldsymbol{r}_{s}, t\right)$ on surface $S$ are known, $\phi\left(\boldsymbol{r}^{\prime}, t^{\prime}\right)$ at any field point $\boldsymbol{r}^{\prime}$ can be computed by using Eq. (15).

\section{TIME DOMAIN BOUNDARY INTEGRAL EQUATION FOR SCATTERING WITH SOLID SURFACES}

A boundary integral equation (BIE) is formed by taking the limit $\boldsymbol{r}^{\prime} \rightarrow \boldsymbol{r}_{s}^{\prime}$ in the integral relation (15), where $\boldsymbol{r}_{s}^{\prime}$ is a point on the boundary. The integral in Eq. (15) involving $\partial G_{0} / \partial \bar{n}$ is weakly singular and, by using Eq. (A1) given in Appendix A (assuming $\boldsymbol{r}_{s}^{\prime}$ is a smooth boundary collocation point), it can be shown that

$$
\begin{aligned}
\lim _{\boldsymbol{r}^{\prime} \rightarrow \boldsymbol{r}_{s}^{\prime}} \int_{S} \frac{\partial G_{0}}{\partial \bar{n}}\left(\boldsymbol{r}_{s}, \boldsymbol{r}^{\prime}\right) \phi\left(\boldsymbol{r}_{s}, t_{R}^{\prime}\right) d \boldsymbol{r}_{s} \\
\quad=\int_{S} \frac{\partial G_{0}}{\partial \bar{n}}\left(\boldsymbol{r}_{s}, \boldsymbol{r}_{s}^{\prime}\right) \phi\left(\boldsymbol{r}_{s}, t_{R}^{\prime}\right) d \boldsymbol{r}_{s}-2 \pi \phi\left(\boldsymbol{r}_{s}^{\prime}, t^{\prime}\right) .
\end{aligned}
$$

Applying this limit to Eq. (15), we get the following time domain boundary integral equation (TDBIE):

$$
\begin{gathered}
2 \pi \phi\left(\boldsymbol{r}_{s}^{\prime}, t^{\prime}\right)-\int_{S}\left(G_{0} \frac{\partial \phi}{\partial \tilde{n}}\left(\boldsymbol{r}_{s}, t_{R}^{\prime}\right)-\frac{\partial G_{0}}{\partial \bar{n}}\left[\phi\left(\boldsymbol{r}_{s}, t_{R}^{\prime}\right)\right.\right. \\
\left.\left.+\frac{\bar{R}}{c \alpha^{2}} \frac{\partial \phi}{\partial t}\left(\boldsymbol{r}_{s}, t_{R}^{\prime}\right)\right]\right) d \boldsymbol{r}_{s}=Q\left(\boldsymbol{r}_{s}^{\prime}, t^{\prime}\right),
\end{gathered}
$$

where $Q\left(\boldsymbol{r}_{s}^{\prime}, t^{\prime}\right)$ denotes the contribution from the external sources to the surface point $\boldsymbol{r}_{s}^{\prime}$ :

$$
Q\left(\boldsymbol{r}_{s}^{\prime}, t^{\prime}\right)=\frac{1}{c^{2}} \int_{V_{s}} \frac{1}{\bar{R}} q\left(\boldsymbol{r}, t_{R}^{\prime}\right) d \boldsymbol{r} .
$$

For sound scattering problems, $\phi\left(\boldsymbol{r}_{s}^{\prime}, t^{\prime}\right)$ on the scattering surface $S$ is to be determined by Eq. (19) when the boundary condition for $\phi$ on $S$ is given. A customary boundary condition on rigid surfaces is that the normal component of the acoustic velocity be zero, i.e., $\boldsymbol{n} \cdot \boldsymbol{u}=0$, which, considering Eq. (1), leads to

$$
\boldsymbol{n} \cdot \nabla \phi=\frac{\partial \phi}{\partial n}\left(\boldsymbol{r}_{s}, t\right)=0, \quad \boldsymbol{r}_{s} \in S .
$$

Indeed, in all the previous literature on wave scattering with a uniform mean flow (e.g., Refs. 1, 3-5, and 22-25), in both the frequency domain and the time domain, boundary conditions of type (21) have been assumed at solid wall boundaries. To implement such a boundary condition, the combined normal derivative appearing in Eq. (19) would then be separated into the normal and tangential components as

$$
\frac{\partial \phi}{\partial \tilde{n}}=\left(1-M_{n}^{2}\right) \frac{\partial \phi}{\partial n}-M_{n}\left(\frac{1}{c} \frac{\partial \phi}{\partial t}+M_{T} \cdot \nabla \phi\right),
$$

where $\boldsymbol{M}_{T}$ is the tangential component of the mean flow Mach number $\boldsymbol{M}$.

In the present paper, however, we propose an alternative boundary condition to be used at solid surfaces when solving TDBIE (19) in the presence of a uniform flow. The new boundary condition is based on a consideration of the acoustic energy.

It can be shown that the convective wave Eq. (2) without the source term has an associated energy equation:

$$
\frac{\partial E}{\partial t}+\nabla \cdot \boldsymbol{J}=0
$$

where

$$
\begin{aligned}
& E=\frac{1}{2}|\nabla \phi|^{2}+\frac{1}{2 c^{2}}\left|\frac{D \phi}{D t}\right|^{2}-\frac{\boldsymbol{U} \cdot \nabla \phi}{c^{2}} \frac{D \phi}{D t}, \\
& \boldsymbol{J}=-\frac{\partial \phi}{\partial t}\left(\nabla \phi-\frac{1}{c^{2}} \frac{D \phi}{D t} \boldsymbol{U}\right), \quad \frac{D}{D t}=\frac{\partial}{\partial t}+\boldsymbol{U} \cdot \nabla .
\end{aligned}
$$

Equation (23) can be validated directly by using the expressions defined in Eq. (24). When substituted by the acoustic velocity and pressure defined in Eq. (1), $\rho_{0} E$ is the usual acoustic energy density in a uniform flow. ${ }^{26-28}$

By Eq. (24), it is immediately clear that the energy flux at a surface of normal $\boldsymbol{n}$ is the following:

$$
J_{n}=\boldsymbol{J} \cdot \boldsymbol{n}=-\frac{\partial \phi}{\partial t}\left(\frac{\partial \phi}{\partial n}-\frac{M_{n}}{c} \frac{D \phi}{D t}\right)=-\frac{\partial \phi}{\partial t} \frac{\partial \phi}{\partial \tilde{n}} .
$$

Clearly, on a surface where the normal component of the mean velocity $M_{n}$ is nonzero, i.e., where the surface is not aligned with the mean flow, application of boundary condition (21) will result in nonzero energy flux, i.e., $J_{n} \neq 0$ and, consequently, cause the surface to be acting like an acoustic energy source or sink according to Eq. (25). This will apparently lead to nonconservation of the total acoustic energy.

Alternatively, the boundary condition on the solid surface may be defined by the requirement that no energy flows into or out of the surface. By Eq. (25) and to ensure energy flux $J_{n}=0$ on solid surfaces, we propose that the boundary condition be modified such that the combined normal derivative of $\phi$, defined in Eq. (12), is zero:

$$
\frac{\partial \phi}{\partial \tilde{n}}\left(\boldsymbol{r}_{s}, t\right)=\frac{\partial \phi}{\partial n}-\frac{M_{n}}{c} \frac{D \phi}{D t}=0, \quad \boldsymbol{r}_{s} \in S
$$

The total acoustic energy will be conserved under this new condition. Equation (26) will be referred to as the zero energy flux (ZEF) boundary condition. 
Now by applying ZEF boundary condition Eq. (26) to Eq. (19), a new formulation of the TDBIE for $\phi\left(\boldsymbol{r}_{s}^{\prime}, t^{\prime}\right)$ with solid surfaces is found as follows:

$$
\begin{aligned}
& 2 \pi \phi\left(\boldsymbol{r}_{s}^{\prime}, t^{\prime}\right)+\int_{S} \frac{\partial G_{0}}{\partial \bar{n}}\left(\phi\left(\boldsymbol{r}_{s}, t_{R}^{\prime}\right)+\frac{\bar{R}}{c \alpha^{2}} \frac{\partial \phi}{\partial t}\left(\boldsymbol{r}_{s}, t_{R}^{\prime}\right)\right) \\
& \quad \times d \boldsymbol{r}_{s}=Q\left(\boldsymbol{r}_{s}^{\prime}, t^{\prime}\right) .
\end{aligned}
$$

Equation (27) is one of the main results of the present paper. It is a new formulation for the time domain boundary integral equation for acoustic scattering by rigid surfaces in a constant mean flow. It is different from those in the literature in several aspects. First, the boundary condition used for Eq. (27) is one that is based on the acoustic energy flux consideration instead of the acoustic normal velocity. The two approaches differ on the part of the boundary where the mean flow itself does not satisfy the slip boundary condition. Second, the new equation is much simpler than those of the previous formulations in which tangential derivatives of the solution on the scattering surface are required to be kept as part of the integral equation. Of course, boundary condition (26) reduces to the usual one [Eq. (21)] wherever the mean flow satisfies the solid wall boundary condition, i.e., $M_{n}=0$.

\section{BURTON-MILLER TYPE REFORMULATION IN TIME DOMAIN WITH A MEAN FLOW}

Direct solution of boundary integral equations for exterior scattering problems, however, is known to suffer numerical instabilities. The instability is generally attributed to the existence of resonance frequencies for the interior domain. ${ }^{1,2,7-10}$ In time domain solutions, the instability is more easily triggered because a continuous spectrum of frequencies within the numerical resolution are present in computation. This instability is one of the major difficulties that have hindered the use of time domain integral equations. Recently, the Burton-Miller type reformulation that has been widely used for exterior scattering problems in the frequency domain has shown to be effective in eliminating the instability in the time domain as well. ${ }^{2,7,8}$ In Ref. 8, a theoretical justification has been provided for the extension of the Burton-Miller formulation to the time domain for the wave equation without flow. In this section, we derive the Burton-Miller reformulation for the TDBIE (27). An analysis on its stability similar to that in Ref. 8 is given in Sec. V.

For convenience of discussion, we define the following time domain double layer potential:

$$
\begin{aligned}
\mathcal{D}[\phi]\left(\boldsymbol{r}^{\prime}, t^{\prime}\right) & =\int_{0}^{t^{+}} \int_{S} \frac{\partial \tilde{G}}{\partial \tilde{n}}\left(\boldsymbol{r}_{s}, t ; \boldsymbol{r}^{\prime}, t^{\prime}\right) \phi\left(\boldsymbol{r}_{s}, t\right) d \boldsymbol{r}_{s} d t \\
& =\int_{S} \frac{\partial G_{0}}{\partial \bar{n}}\left(\boldsymbol{r}_{s}, \boldsymbol{r}^{\prime}\right)\left(\phi\left(\boldsymbol{r}_{s}, t_{R}^{\prime}\right)+\frac{\bar{R}}{c \alpha^{2}} \frac{\partial \phi}{\partial t}\left(\boldsymbol{r}_{s}, t_{R}^{\prime}\right)\right) d \boldsymbol{r}_{s} .
\end{aligned}
$$

The Burton-Miller type reformulation is carried out by applying a linear combination of the time and certain normal derivatives to the time domain integral equation. In earlier studies of the Burton-Miller formulation for scattering with a flow, the modified normal derivative (10) had been used., ${ }^{1,4}$ Here, we propose that the normal derivative to be used for the Burton-Miller formulation be the combined normal derivative defined in Eq. (12). Specifically, the Burton-Miller reformulation is obtained by applying the following derivative operator to the boundary integral equation at surface points $\boldsymbol{r}_{s}^{\prime}$ :

$$
\tilde{a} \frac{\partial}{\partial t^{\prime}}+\tilde{b} c \frac{\partial}{\partial \tilde{n}^{\prime}},
$$

where $\tilde{a}$ and $\tilde{b}$ are constants and $c$ is the speed of sound. That is, operator (29) is applied to the integral Eq. (27) to give

$$
\begin{aligned}
& \tilde{a} \frac{\partial}{\partial t^{\prime}}\left(2 \pi \phi\left(\boldsymbol{r}_{s}^{\prime}, t^{\prime}\right)+\mathcal{D}[\phi]\left(\boldsymbol{r}_{s}^{\prime}, t^{\prime}\right)\right) \\
& \quad+\left.\tilde{b} c \frac{\partial}{\partial \tilde{n}^{\prime}}\left(4 \pi \phi\left(\boldsymbol{r}^{\prime}, t^{\prime}\right)+\mathcal{D}[\phi]\left(\boldsymbol{r}^{\prime}, t^{\prime}\right)\right)\right|_{\boldsymbol{r}^{\prime}=\boldsymbol{r}_{s}^{\prime}} \\
& \quad=\tilde{a} \frac{\partial Q}{\partial t^{\prime}}\left(\boldsymbol{r}_{s}^{\prime}, t^{\prime}\right)+\tilde{b} c \frac{\partial Q}{\partial \tilde{n}^{\prime}}\left(\boldsymbol{r}_{s}^{\prime}, t^{\prime}\right) .
\end{aligned}
$$

Applying again the ZEF boundary condition (26), Eq. (30) is expanded to be the following:

$$
\begin{aligned}
\tilde{a}[2 & 2 \pi \frac{\partial \phi}{\partial t}\left(\boldsymbol{r}_{s}^{\prime}, t^{\prime}\right)+\int_{S} \frac{\partial G_{0}}{\partial \bar{n}}\left(\boldsymbol{r}_{s}, \boldsymbol{r}^{\prime}\right)\left(\frac{\partial \phi}{\partial t}\left(\boldsymbol{r}_{s}, t_{R}^{\prime}\right)\right. \\
& \left.\left.+\frac{\bar{R}}{c \alpha^{2}} \frac{\partial \phi}{\partial t^{2}}\left(\boldsymbol{r}_{s}, t_{R}^{\prime}\right)\right) d \boldsymbol{r}_{s}\right]+\tilde{b} c\left[\frac{\partial}{\partial \tilde{n}^{\prime}} \int_{S} \frac{\partial G_{0}}{\partial \bar{n}}\left(\boldsymbol{r}_{s}, \boldsymbol{r}^{\prime}\right)\right. \\
& \left.\times\left(\phi\left(\boldsymbol{r}_{s}, t_{R}^{\prime}\right)+\frac{\bar{R}}{c \alpha^{2}} \frac{\partial \phi}{\partial t}\left(\boldsymbol{r}_{s}, t_{R}^{\prime}\right)\right) d \boldsymbol{r}_{s}\right]_{\boldsymbol{r}^{\prime}=\boldsymbol{r}_{s}^{\prime}} \\
= & \tilde{a} \frac{\partial Q}{\partial t^{\prime}}\left(\boldsymbol{r}_{s}^{\prime}, t^{\prime}\right)+\tilde{b} c \frac{\partial Q}{\partial \tilde{n}^{\prime}}\left(\boldsymbol{r}_{s}^{\prime}, t^{\prime}\right) .
\end{aligned}
$$

Note that an integral with a kernel $\left(\partial^{2} G_{0} / \partial \bar{n}^{\prime} \partial \bar{n}\right)\left(\boldsymbol{r}_{s}, \boldsymbol{r}_{s}^{\prime}\right)$ is hyper-singular when $\boldsymbol{r}_{s}$ coincides with $\boldsymbol{r}_{\boldsymbol{s}}^{\prime}$. In particular, we have

$$
\begin{aligned}
\frac{\partial^{2} G_{0}}{\partial \bar{n}^{\prime} \partial \bar{n}}\left(\boldsymbol{r}_{s}, \boldsymbol{r}_{s}^{\prime}\right)= & \frac{\partial}{\partial \bar{n}^{\prime}}\left[-\alpha^{2} \frac{\boldsymbol{n} \cdot\left(\boldsymbol{r}_{s}-\boldsymbol{r}_{s}^{\prime}\right)}{\bar{R}^{3}}\right]=\frac{\alpha^{2}}{\bar{R}^{3}}\left[\boldsymbol{n} \cdot \boldsymbol{n}^{\prime}-M_{n^{\prime}} M_{n}\right] \\
& +3 \alpha^{4} \frac{\left.\boldsymbol{n} \cdot\left(\boldsymbol{r}_{s}-\boldsymbol{r}_{s}^{\prime}\right)\right]\left[\boldsymbol{n}^{\prime} \cdot\left(\boldsymbol{r}_{s}^{\prime}-\boldsymbol{r}_{s}\right)\right]}{\bar{R}^{5}}
\end{aligned}
$$

Thus, $\left(\partial^{2} G_{0} / \partial \bar{n}^{\prime} \partial \bar{n}\right)\left(\boldsymbol{r}_{s}, \boldsymbol{r}_{s}^{\prime}\right)$ is of order $O\left(1 /\left|\boldsymbol{r}_{s}-\boldsymbol{r}_{s}^{\prime}\right|^{3}\right)$ as $\boldsymbol{r}_{s} \rightarrow \boldsymbol{r}_{s}^{\prime}$.

We consider the following regularization process for the hyper-singular integral in Eq. (31) that adds and subtracts a term involving the value at the collocation point $\phi\left(\boldsymbol{r}_{s}^{\prime}, t^{\prime}\right)$ :

$$
\begin{aligned}
& \frac{\partial}{\partial \tilde{n}^{\prime}}\left[\int_{S} \frac{\partial G_{0}}{\partial \bar{n}}\left(\boldsymbol{r}_{s}, \boldsymbol{r}_{s}^{\prime}\right)\left(\phi\left(\boldsymbol{r}_{s}, t_{R}^{\prime}\right)+\frac{\bar{R}}{c \alpha^{2}} \frac{\partial \phi}{\partial t}\left(\boldsymbol{r}_{s}, t_{R}^{\prime}\right)\right) d \boldsymbol{r}_{s}\right] \\
& =\frac{\partial}{\partial \tilde{n}^{\prime}}\left[\int _ { S } \frac { \partial G _ { 0 } } { \partial \overline { n } } ( \boldsymbol { r } _ { s } , \boldsymbol { r } _ { s } ^ { \prime } ) \left(\phi\left(\boldsymbol{r}_{s}, t_{R}^{\prime}\right)-\phi\left(\boldsymbol{r}_{s}^{\prime}, t^{\prime}\right)+\frac{\bar{R}}{c \alpha^{2}} \frac{\partial \phi}{\partial t}\right.\right. \\
& \left.\left.\quad \times\left(\boldsymbol{r}_{s}, t_{R}^{\prime}\right)\right) d \boldsymbol{r}_{s}\right]+\phi\left(\boldsymbol{r}_{s}^{\prime}, t^{\prime}\right) \frac{\partial}{\partial \tilde{n}^{\prime}}\left[\int_{S} \frac{\partial G_{0}}{\partial \bar{n}}\left(\boldsymbol{r}_{s}, \boldsymbol{r}_{s}^{\prime}\right) d \boldsymbol{r}_{s}\right] .
\end{aligned}
$$

The first integral is now integrable by Cauchy Principal Value (Appendix B) and the second integral is zero according to Eq. (A1) given in Appendix A. Upon carrying out the 
derivatives inside the first integral shown above, we get the following Burton-Miller reformulation of the time domain boundary integral equation (BM-TDBIE):

$$
\begin{aligned}
& 2 \pi \tilde{a} \frac{\partial \phi\left(\boldsymbol{r}_{s}^{\prime}, t^{\prime}\right)}{\partial t}+\tilde{a} \int_{S} \frac{\partial G_{0}}{\partial \bar{n}}\left(\frac{\partial \phi}{\partial t}\left(\boldsymbol{r}_{s}, t_{R}^{\prime}\right)\right. \\
& \left.+\frac{\bar{R}}{c \alpha^{2}} \frac{\partial^{2} \phi}{\partial t^{2}}\left(\boldsymbol{r}_{s}, t_{R}^{\prime}\right)\right) d \boldsymbol{r}_{s}-\frac{\tilde{b}}{c \alpha^{4}} \int_{S} \bar{R}^{3} \frac{\partial G_{0}}{\partial \bar{n}^{\prime}} \frac{\partial G_{0}}{\partial \bar{n}} \frac{\partial^{2} \phi}{\partial t^{2}}\left(\boldsymbol{r}_{s}, t_{R}^{\prime}\right) d \boldsymbol{r}_{s} \\
& +\tilde{b} c \int_{S} \frac{\partial^{2} G_{0}}{\partial \bar{n}^{\prime} \partial \bar{n}}\left(\phi\left(\boldsymbol{r}_{s}, t_{R}^{\prime}\right)-\phi\left(\boldsymbol{r}_{s}^{\prime}, t^{\prime}\right)+\frac{\bar{R}}{c \alpha^{2}} \frac{\partial \phi}{\partial t}\left(\boldsymbol{r}_{s}, t_{R}^{\prime}\right)\right) d \boldsymbol{r}_{s} \\
& =\tilde{a} \frac{\partial Q}{\partial t^{\prime}}\left(\boldsymbol{r}_{s}^{\prime}, t^{\prime}\right)+\tilde{b} c \frac{\partial Q}{\partial \tilde{n}^{\prime}}\left(\boldsymbol{r}_{s}^{\prime}, t^{\prime}\right) .
\end{aligned}
$$

The proper values for the coefficients $\tilde{a}$ and $\tilde{b}$ will be given in Sec. V where stability of Eq. (34) will be discussed.

\section{STABILITY OF THE TIME DOMAIN BURTON-MILLER FORMULATION IN THE PRESENCE OF A MEAN FLOW}

Following closely the work in Ref. 8 for the case without flow, we demonstrate in this section that the Burton-Miller type reformulation presented in Sec. IV eliminates the nontrivial solutions of the homogeneous integral equation in the case with a flow as well.

Suppose that there is a nontrivial solution $\phi_{0}\left(\boldsymbol{r}_{s}, t\right)$ to the homogeneous formulation for Eq. (34) in which the source term is set to zero. We will show in what follows that such a solution is not possible. Consider the double layer potential Eq. (28) extended to domains both exterior and interior of surface $S$ :

$$
\begin{aligned}
& \mathcal{D}\left[\phi_{0}\right]\left(\boldsymbol{r}^{\prime}, t^{\prime}\right) \\
& =\int_{S} \frac{\partial G_{0}}{\partial \bar{n}}\left(\boldsymbol{r}_{s}, \boldsymbol{r}^{\prime}\right)\left(\phi_{0}\left(\boldsymbol{r}_{s}, t_{R}^{\prime}\right)+\frac{\bar{R}}{c \alpha^{2}} \frac{\partial \phi_{0}}{\partial t}\left(\boldsymbol{r}_{s}, t_{R}^{\prime}\right)\right) d \boldsymbol{r}_{s} \\
& \equiv\left\{\begin{array}{l}
w^{+}, \boldsymbol{r}^{\prime} \in V, \text { exterior of } S \\
w_{0}, \boldsymbol{r}^{\prime}=\boldsymbol{r}_{s}^{\prime} \text { on } \mathrm{S} \\
w^{-}, \boldsymbol{r}^{\prime} \in V^{-}, \text {interior of } S .
\end{array}\right.
\end{aligned}
$$

We note that $w^{+}$and $w^{-}$satisfy the homogeneous convective wave equation in the exterior and interior domains of $S$, respectively. It can also be shown that

$$
\begin{aligned}
& \lim _{r^{\prime} \rightarrow r_{s}^{\prime}} w^{+}=w_{0}-2 \pi \phi_{0}\left(r_{s}^{\prime}, t^{\prime}\right), \\
& \lim _{r^{\prime} \rightarrow r_{s}^{\prime}} w^{-}=w_{0}+2 \pi \phi_{0}\left(\boldsymbol{r}_{s}^{\prime}, t^{\prime}\right), \\
& \lim _{r^{\prime} \rightarrow r_{s}^{\prime}} \frac{\partial w^{+}}{\partial \tilde{n}^{\prime}}=\lim _{r^{\prime} \rightarrow r_{s}^{\prime}} \frac{\partial w^{-}}{\partial \tilde{n}^{\prime}} .
\end{aligned}
$$

Equations (35) and (36) can be found by using the limits given in Eq. (A1) in Appendix A, and Eq. (37) follows after an application of the regularization process (33) to both sides of the equation.

Now since $\phi_{0}\left(\boldsymbol{r}_{s}, t\right)$ satisfies the homogeneous Burton-Miller formulation for Eq. (30) where the right hand side is zero, we have, at $\boldsymbol{r}^{\prime}=\boldsymbol{r}_{s}^{\prime}$,

$$
\tilde{a} \frac{\partial}{\partial t^{\prime}}\left(2 \pi \phi_{0}+w_{0}\right)+\left.\tilde{b} c \frac{\partial}{\partial \tilde{n}^{\prime}}\left(4 \pi \phi_{0}+w^{+}\right)\right|_{r_{s}^{\prime}}=0 .
$$

By the jump conditions (35)-(37) as well as the ZEF boundary condition (26), the above yields

$$
\tilde{a} \frac{\partial w^{-}}{\partial t^{\prime}}+\tilde{b} c \frac{\partial w^{-}}{\partial \tilde{n}^{\prime}}=0
$$

On the other hand, since $w^{-}$satisfies the convective wave equation and by the energy Eq. (23) of the convective wave equation, we have

$$
\begin{gathered}
\frac{\partial}{\partial t} \int_{V^{-}}\left[\frac{1}{2}\left|\nabla w^{-}\right|^{2}+\frac{1}{2 c^{2}}\left|\frac{D w^{-}}{D t}\right|^{2}-\frac{\boldsymbol{U} \cdot \nabla w^{-}}{c^{2}} \frac{D w^{-}}{D t}\right] d \boldsymbol{r} \\
=\int_{V^{-}} \nabla \cdot\left[\frac{\partial w^{-}}{\partial t}\left(\nabla w^{-}-\frac{1}{c^{2}} \frac{D w^{-}}{D t} \boldsymbol{U}\right)\right] d \boldsymbol{r},
\end{gathered}
$$

which, with an application of the divergence theorem, becomes

$$
\begin{aligned}
& \int_{V^{-}} {\left[\frac{1}{2}\left|\nabla w^{-}\right|^{2}+\frac{1}{2 c^{2}}\left|\frac{D w^{-}}{D t}\right|^{2}-\frac{\boldsymbol{U} \cdot \nabla w^{-}}{c^{2}} \frac{D w^{-}}{D t}\right] d \boldsymbol{r} } \\
&=-\int_{0}^{t^{+}} \int_{S} \frac{\partial w^{-}}{\partial t} \frac{\partial w^{-}}{\partial \tilde{n}} d \boldsymbol{r}_{s} d t
\end{aligned}
$$

where $V^{-}$represents the volume interior of $S$. The minus sign on the right hand side has been added due to the fact that the normal derivative used in Eq. (39) is still the one that is inward of the body surface. Note that, for subsonic flows where $|\boldsymbol{U}|<c$, the left hand side of Eq. (39) is nonnegative:

$$
\begin{aligned}
\frac{1}{2}\left|\nabla w^{-}\right|^{2}+\frac{1}{2 c^{2}}\left|\frac{D w^{-}}{D t}\right|^{2}-\frac{\boldsymbol{U} \cdot \nabla w^{-}}{c^{2}} \frac{D w^{-}}{D t} \\
=\frac{1}{2}\left(\left|\nabla w^{-}\right|-\frac{1}{c}\left|\frac{D w^{-}}{D t}\right|\right)^{2}+\frac{1}{c}\left|\nabla w^{-}\right|\left|\frac{D w^{-}}{D t}\right| \\
\quad-\frac{\boldsymbol{U} \cdot \nabla w^{-}}{c^{2}} \frac{D w^{-}}{D t} \geq 0 .
\end{aligned}
$$

On the other hand, using Eq. (38), the right hand side of Eq. (39) will be nonpositive:

$$
-\int_{0}^{t^{+}} \int_{S} \frac{\partial w^{-}}{\partial t} \frac{\partial w^{-}}{\partial \tilde{n}} d \boldsymbol{r}_{s}=\frac{1}{c^{2}} \int_{0}^{t^{+}} \int_{S} \frac{\tilde{a}}{\tilde{b} c}\left|\frac{\partial w^{-}}{\partial t}\right|^{2} d \boldsymbol{r}_{s} \leq 0,
$$

provided that

$$
\frac{\tilde{a}}{\tilde{b}}<0 .
$$

The above implies that $w^{-}$has to be a trivial solution, i.e., $w^{-} \equiv 0$ under condition (40). A simple choice for $\tilde{a}$ and $\tilde{b}$ is $\tilde{a}=-\tilde{b}=1$.

As shown in Refs. 8-10 and mentioned in Sec. IV, numerical instability associated with solving TDBIE is attributed to the existence of nontrivial resonant solutions. The 
analysis in this section shows that nontrivial solutions of the homogeneous integral equation are eliminated by the Burton-Miller reformulation of TDBIE (27). Hence, the instability caused by the resonant solutions will be effectively suppressed by using BM-TDBIE (34) under condition (40).

\section{TIME DOMAIN BOUNDARY ELEMENT METHOD}

In this section and Sec. VII, we describe a numerical solution of Eq. (34) by the time domain boundary element method (TDBEM) and demonstrate numerical stability of the new formulation.

Let surface $S$ be discretized by surface elements $E_{j}$, $j=1,2, \ldots, N_{e}$, where $N_{e}$ is the total number of elements, and the time be discretized by $t_{n}=n \Delta t$, where $\Delta t$ is the time step. The time domain numerical solution on the discretized surface can be expanded as

$$
\phi\left(\boldsymbol{r}_{s}, t\right)=\sum_{n=0}^{N_{t}} \sum_{j=1}^{N_{e}} u_{j}^{n} \varphi_{j}\left(\boldsymbol{r}_{s}\right) \psi_{n}(t),
$$

where $\varphi_{j}\left(\boldsymbol{r}_{S}\right)$ is the surface basis function for element $E_{j}$ and $\psi_{n}(t)$ is the temporal basis function for time node $t_{n}$. Here $N_{t}$ is the total number of time steps. For simplicity, we consider only constant elements where collocation node $\boldsymbol{r}_{j}$ for $E_{j}$ is located at the center of the element and the nodal basis function is

$$
\varphi_{j}\left(\boldsymbol{r}_{s}\right)= \begin{cases}1, & \boldsymbol{r}_{s} \text { on element } E_{j} \text { that contains node } \boldsymbol{r}_{j} \\ 0, & \text { otherwise. }\end{cases}
$$

The temporal basis function is taken to be the thirdorder shifted Lagrange basis polynomial that is commonly used for time domain boundary element methods: ${ }^{11,29}$

$$
\psi_{n}(t)=\Psi\left(\frac{t-t_{n}}{\Delta t}\right)
$$

where

$$
\Psi(\tau)= \begin{cases}1+\frac{11}{6} \tau+\tau^{2}+\frac{1}{6} \tau^{3}, & -1<\tau \leq 0 \\ 1+\frac{1}{2} \tau-\tau^{2}-\frac{1}{2} \tau^{3}, & 0<\tau \leq 1 \\ 1-\frac{1}{2} \tau-\tau^{2}+\frac{1}{2} \tau^{3}, & 1<\tau \leq 2 \\ 1-\frac{11}{6} \tau+\tau^{2}-\frac{1}{6} \tau^{3}, & 2<\tau \leq 3 \\ 0, & \text { other. }\end{cases}
$$

For example, at any point $\boldsymbol{r}_{s}$ on element $E_{j}$ and at any off-nodal time $t=t_{n}-\eta \Delta t, 0 \leq \eta<1$, the value for $\phi\left(\boldsymbol{r}_{s}, t\right)$ is found by

$$
\begin{aligned}
\phi\left(\boldsymbol{r}_{s}, t\right)= & \varphi_{j}\left(\boldsymbol{r}_{s}\right)\left[u_{j}^{n} \Psi(-\eta)+u_{j}^{n-1} \Psi(1-\eta)\right. \\
& \left.+u_{j}^{n-2} \Psi(2-\eta)+u_{j}^{n-3} \Psi(3-\eta)\right] .
\end{aligned}
$$

With the nodal spatial and temporal basis functions defined above, expansion coefficient $u_{j}^{n}$ in Eq. (41) represents the value of $\phi$ at the collocation node $\boldsymbol{r}_{j}$ on element $E_{j}$ at time level $t_{n}$. By substituting expansion Eq. (41) into BM-TDBIE (34) and evaluating the equation at collocation points $\boldsymbol{r}_{i}$ of all elements, $i=1,2, \ldots, N_{e}$, and at time level $t_{n}$, a march-on-in-time scheme (MOT) is obtained that can be expressed in a matrix form as

$$
\boldsymbol{B}_{0} \boldsymbol{u}^{n}=\boldsymbol{q}^{n}-\boldsymbol{B}_{1} \boldsymbol{u}^{n-1}-\boldsymbol{B}_{2} \boldsymbol{u}^{n-2}-\cdots-\boldsymbol{B}_{J} \boldsymbol{u}^{n-J},
$$

where $\boldsymbol{u}^{k}$ denotes a vector that contains all the expansion coefficients $\left\{u_{j}^{k}, j=1,2, \ldots, N_{e}\right\}$ at time level $t_{k}$. The nonzero entries for matrices $\boldsymbol{B}_{k}, k=0,1,2, ., J$, in Eq. (46) can be found to be

$$
\begin{aligned}
\left\{\boldsymbol{B}_{k}\right\}_{i j}= & 2 \pi \tilde{a} \delta_{i j} \psi_{n-k}^{\prime}\left(t_{n}\right)+\tilde{a} \int_{E_{j}} \frac{\partial G_{0}}{\partial \bar{n}}\left(\psi_{n-k}^{\prime}\left(t_{R}^{n}\right)\right. \\
& \left.+\frac{\bar{R}}{c \alpha^{2}} \psi_{n-k}^{\prime \prime}\left(t_{R}^{n}\right)\right) d \boldsymbol{r}_{s}+\tilde{b} c \delta_{i j} \delta_{k 0} D_{i}+\tilde{b} c \int_{E_{j}} \frac{\partial^{2} G_{0}}{\partial \bar{n}^{\prime} \partial \bar{n}} \\
& \times\left(\psi_{n-k}\left(t_{R}^{n}\right)-\delta_{i j} \psi_{n-k}\left(t_{n}\right)+\frac{\bar{R}}{c \alpha^{2}} \psi_{n-k}^{\prime}\left(t_{R}^{n}\right)\right) d \boldsymbol{r}_{s} \\
& +\frac{\tilde{b}}{c \alpha^{4}} \int_{E_{j}} \bar{R}^{3} \frac{\partial G_{0}}{\partial \bar{n}^{\prime}} \frac{\partial G_{0}}{\partial \bar{n}} \psi_{n-k}^{\prime \prime}\left(t_{R}^{n}\right) d \boldsymbol{r}_{s},
\end{aligned}
$$

for $i, j=1,2, \ldots, N_{e}$, where $\delta_{i j}$ and $\delta_{k 0}$ are Kronecker delta functions and a prime in the above denotes derivative with respect to time, and

$$
\begin{aligned}
t_{R}^{n} & =t_{n}+\boldsymbol{\beta} \cdot\left(\boldsymbol{r}_{i}-\boldsymbol{r}_{s}\right)-\frac{\bar{R}\left(\boldsymbol{r}_{s}, \boldsymbol{r}_{i}\right)}{c \alpha^{2}}, \\
D_{i} & =-\int_{S-E_{i}} \frac{\partial^{2} G_{0}}{\partial \bar{n}^{\prime} \partial \bar{n}}\left(\boldsymbol{r}_{s}, \boldsymbol{r}_{i}\right) d \boldsymbol{r}_{s} .
\end{aligned}
$$

It is easy to see that the entry $\left\{\boldsymbol{B}_{k}\right\}_{i j}$ represents contributions to the value at node $\boldsymbol{r}_{i}$ and time $t_{n}$ from nodal value of element $E_{j}$ of time level $t_{n-k}$. The integrals in Eq. (47) are to be evaluated using high-order quadrature on each element. For the computational results reported in this paper, each element is mapped to a standard element of $[-1,1] \times[-1,1]$ and Legendre-Gauss quadrature rule of degree six is used for integration in each dimension. Integration on the singular elements where $i=j$ is detailed in Appendix B.

The index $J$ in Eq. (46) denotes the maximum time history of the solution required for Eq. (46) and is dependent on the length of the scattering surface and the mean flow as

$$
J=\frac{\bar{L}}{c \alpha^{2} \Delta t}+3, \quad \bar{L}=\max _{\boldsymbol{r}_{s}, \boldsymbol{r}_{s}^{\prime} \in S}\left[-\boldsymbol{M} \cdot\left(\boldsymbol{r}_{s}^{\prime}-\boldsymbol{r}_{s}\right)+\bar{R}\left(\boldsymbol{r}_{s}, \boldsymbol{r}_{s}^{\prime}\right)\right] .
$$

Due to the limited temporal stencil width shown in Eqs. (44) and (45), the $\boldsymbol{B}$ matrices are sparse. In particular, we note that matrix $\boldsymbol{B}_{0}$ in Eq. (46) is a very sparse matrix and represents interactions within the same element or between nearby nodes at the same time level $t_{n}$. $\boldsymbol{B}_{0}$ is also found to be diagonally dominant. Solutions for $\boldsymbol{u}^{n}$ in Eq. (46) can be found efficiently by an iterative method, such as the Jacobi iterative method, with rapid convergence. ${ }^{11,30}$ 


\section{EIGENVALUE STABILITY ANALYSIS OF THE NEW INTEGRAL EQUATION}

As mentioned in Secs. V and VI, direct numerical solution of the time domain boundary integral Eq. (27) is prone to numerical instabilities. In Fig. 2, we first show an example of scattering of a point source by a parabolic wing in a mean flow of Mach number $0.5, \boldsymbol{M}=(0.5,0,0)$, to demonstrate the elimination of numerical instability by the Burton-Miller reformulation of TDBIE (27). The geometry of the scattering surface is a convex parabolic wing and is defined as follows:

$$
z=0.1 L_{x}\left(1-x^{2} / L_{x}^{2}\right),-L_{x} \leq x \leq L_{x}, \quad-L_{y} \leq y \leq L_{y},
$$

where $L_{x}=L_{y}=0.5$. In this example, the scattering surface is discretized by 2316 quadrilateral elements. The source function is a broadband point source defined as the following:

$$
q(\boldsymbol{r}, t)=e^{-\sigma t^{2}} \delta\left(\boldsymbol{r}-\boldsymbol{r}_{0}\right),
$$

where $\boldsymbol{r}_{0}=(0,0,1)$ and $\sigma=1.42 /(6 \Delta t)^{2}$.

The time history of the solution on a surface collocation point is plotted in Fig. 2 for the cases without and with Burton-Miller reformulation. The top figure shows the result obtained by directly solving the TDBIE (27). It is seen that the solution initially behaves well but eventually becomes unstable. On the other hand, the solution obtained by the BM-TDBIE (34), shown in the bottom figure, remains stable.

To further study the stability of the MOT scheme (46), we conduct a numerical eigenvalue study of the discretized system of equations. ${ }^{31}$ For numerical stability considerations, we look for solutions of the form

$$
\boldsymbol{u}^{n}=\lambda^{n} \boldsymbol{e}_{0}
$$
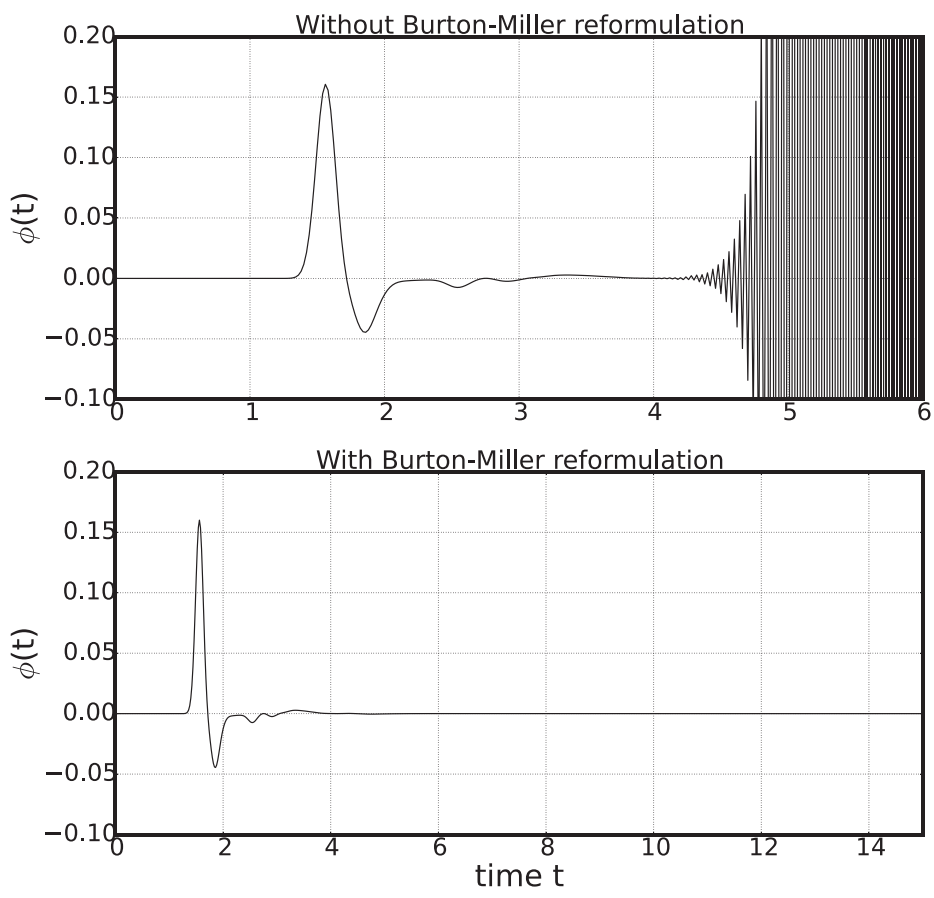

to the corresponding homogeneous system for Eq. (46). By substituting Eq. (52) into Eq. (46) without the source term, we obtain a polynomial eigenvalue problem

$$
\left[\boldsymbol{B}_{0} \lambda^{J}+\boldsymbol{B}_{1} \lambda^{J-1}+\boldsymbol{B}_{2} \lambda^{J-2}+\cdots+\boldsymbol{B}_{J-1} \lambda+\boldsymbol{B}_{J}\right] \boldsymbol{e}_{0}=0,
$$

which can be cast into a generalized eigenvalue problem as follows:

$$
\begin{gathered}
{\left[\begin{array}{ccccc}
-\boldsymbol{B}_{1} & -\boldsymbol{B}_{2} & \cdots & -\boldsymbol{B}_{J-1} & -\boldsymbol{B}_{J} \\
\boldsymbol{I} & 0 & \cdots & 0 & 0 \\
0 & \boldsymbol{I} & \cdots & 0 & 0 \\
\cdots & \cdots & \cdots & \cdots & \cdots \\
0 & 0 & \cdots & 0 & 0 \\
0 & 0 & \cdots & \boldsymbol{I} & 0
\end{array}\right]\left[\begin{array}{c}
\boldsymbol{e}_{J-1} \\
\boldsymbol{e}_{J-2} \\
\vdots \\
\vdots \\
\boldsymbol{e}_{1} \\
\boldsymbol{e}_{0}
\end{array}\right]} \\
=\lambda\left[\begin{array}{cccccc}
\boldsymbol{B}_{0} & 0 & 0 & \cdots & 0 & 0 \\
0 & \boldsymbol{I} & 0 & \cdots & 0 & 0 \\
0 & 0 & \boldsymbol{I} & \cdots & 0 & 0 \\
\cdots & \cdots & \cdots & \cdots & \cdots & \cdots \\
0 & 0 & 0 & \cdots & \boldsymbol{I} & 0 \\
0 & 0 & 0 & \cdots & 0 & \boldsymbol{I}
\end{array}\right]\left[\begin{array}{c}
\boldsymbol{e}_{J-1} \\
\boldsymbol{e}_{J-2} \\
\vdots \\
\vdots \\
\boldsymbol{e}_{1} \\
\boldsymbol{e}_{0}
\end{array}\right],
\end{gathered}
$$

where $\boldsymbol{e}_{j}=\lambda^{j} \boldsymbol{e}_{0}$. For numerical scheme (46) to be stable, it is necessary that $|\lambda| \leq 1$ for all eigenvalues of Eq. (54). We note that this is a necessary but not sufficient condition for stability because the iteration matrix for Eq. (54) is not a normal matrix. ${ }^{32}$

Eigenvalue analyses of scattering by two geometric shapes are presented in Table I. One of the geometries is the parabolic wing as described previously in Eq. (50). The other is a sphere of radius $a=0.5$. The surface of the sphere is first discretized by 512 unstructured triangular elements each of which is then subdivided into three quadrilateral surface elements resulting in a total of 1536 surface elements. The mean flow Mach number varies from 0 to 0.9. A total of eight cases are considered in Table I.
FIG. 2. Time history of numerical solution on a surface collocation point, showing the elimination of instability by Burton-Miller reformulation of TDBIE. $\boldsymbol{M}=(0.5,0,0)$. The nondimensional time step is $c \Delta t / L_{x}=0.04$. Top: solution of Eq. (27) without Burton-Miller reformulation; bottom: solution by BMTDBIE Eq. (34). 
TABLE I. Maximum eigenvalue, $|\lambda|_{\max }$, computed using Eq. (54) for scattering by a parabolic wing and by a sphere, for cases with and without Burton-Miller (B-M) reformulation. $N_{e}$ is the total number of elements and $M$ is the mean flow Mach number. The nondimensional time step is $c \Delta t / L=0.04$ for all the cases where the length scale $L$ is $L_{x}$ and radius $a$, respectively, for the parabolic wing and the sphere.

\begin{tabular}{|c|c|c|c|c|c|c|c|}
\hline \multicolumn{4}{|c|}{ Parabolic wing } & \multicolumn{4}{|c|}{ Sphere } \\
\hline \multicolumn{4}{|c|}{$|\lambda|_{\max }$} & \multicolumn{4}{|c|}{$|\lambda|_{\max }$} \\
\hline$N_{e}$ & $M$ & $\begin{array}{l}\text { with B-M } \\
\text { Eq. (34) }\end{array}$ & $\begin{array}{c}\text { without B-M } \\
\text { Eq. (27) }\end{array}$ & $N_{e}$ & $M$ & $\begin{array}{l}\text { with B-M } \\
\text { Eq. (34) }\end{array}$ & $\begin{array}{c}\text { without B-M } \\
\text { Eq. (27) }\end{array}$ \\
\hline 2316 & 0.0 & 1.000000 & 1.095949 & 1536 & 0.0 & 1.000000 & 1.007840 \\
\hline 2316 & 0.3 & 1.000000 & 1.160628 & 1536 & 0.3 & 1.000000 & 1.000000 \\
\hline 2316 & 0.6 & 1.000000 & 1.129116 & 1536 & 0.6 & 1.000000 & 0.999968 \\
\hline 2316 & 0.9 & 1.000000 & 1.582909 & 1536 & 0.9 & 1.000000 & 1.003901 \\
\hline
\end{tabular}

Eigenvalues of the generalized eigenvalue problem (54) can be found via a sparse eigenvalue solver available in MATLAB and Python, or by a matrix power iteration method detailed in Appendix C. The values of the largest eigenvalue for the eight cases are listed in Table I. For the Burton-Miller formulation BM-TDBIE (34), all eigenvalues are no greater than unity and stability is observed. In contrast, direct solution of Eq. (27) results in eigenvalues greater than unity in all but two of the eight cases studied, indicating that Eq. (27) without Burton-Miller reformulation can lead to unstable solutions.

\section{A NUMERICAL EXAMPLE}

In this section, we show a numerical example of sound scattering by a solid body in the presence of a uniform mean flow. The geometry of the solid body is that of the parabolic wing as defined in Eq. (50). The dimensions of the wing in the current example are $L_{x}=0.5, L_{y}=1.5$. The incident field is produced by a point source for the velocity potential of the form (51), located at $\boldsymbol{r}_{0}=\left(0,0,10 L_{x}\right)$, directly above the center point of the wing. The mean flow is assumed to be in the direction of the $x$ axis, $\boldsymbol{M}=(M, 0,0)$, where $M$ is the flow Mach number. For the results shown in this example, a total of 4364 quadrilateral elements are used for the discretization of the parabolic wing surface. The far field pressure directivity is to be computed as illustrated in the schematics of the computational domain in Fig. 3. The setup of the problem is the same as that considered in Ref. 3. Our computational results will be compared with those in Ref. 3 .

The time domain boundary integral Eq. (34) is first solved by the MOT scheme (46) as described in Sec. VI. After the value of $\phi$ on the scattering surface is found, the solutions at far field points can be computed using Eq. (15) with the ZEF boundary condition (26) applied. From the velocity potential function $\phi(\boldsymbol{r}, t)$, the acoustic pressure $p(\boldsymbol{r}, t)$ is then obtained by the relation given in Eq. (1), where the temporal and spatial derivatives are computed by finite difference approximations. Here, the sixth-order central difference is used. Finally, for any selected frequency $\omega$, the frequency domain solution can be obtained from the time domain results by either using the fast Fourier transform algorithm or the following summation:

$$
\begin{aligned}
p(\boldsymbol{r}, \omega)= & \Delta t\left[p\left(\boldsymbol{r}, t_{1}\right) e^{-i \omega t_{1}}+p\left(\boldsymbol{r}, t_{2}\right) e^{-i \omega t_{2}}+p\left(\boldsymbol{r}, t_{3}\right) e^{-i \omega t_{3}}\right. \\
& \left.+\cdots+p\left(\boldsymbol{r}, t_{N_{t}}\right) e^{-i \omega t_{N_{t}}}\right]
\end{aligned}
$$

where $\Delta t$ is the time step of the MOT scheme and $N_{t}$ is the total number of time steps.

To compare with the results presented in Ref. 3, far field pressure directivity is calculated at three frequencies: $k L_{x}=1,3$, and 5 , where $k=\omega / c$ is the wave-number. A value of nondimensional time step $c \Delta t / L_{x}=0.05$ is used in the computation, which yields a resolution of approximately $25 \Delta t$ per period of the highest frequency $k L_{x}=5$, sufficiently fine for the third-order time basis function (44) used for the example. $^{29}$

As in Ref. 3, the directivity function $D(\theta)$ is defined as

$$
D(\theta)=\frac{\hat{R}}{L_{x}}\left|\frac{p(\hat{\boldsymbol{r}}, \omega)}{p_{0}(\omega)}\right|,
$$

where the far field points are sampled on a circle of radius $\hat{R}$ on the $x-z$ plane across the mid-span of the parabolic wing:

$$
\hat{\boldsymbol{r}}=(\hat{R} \cos \theta, 0, \hat{R} \sin \theta),
$$

with $\hat{R}=105 L_{x}$ as was used in Ref. 3. In Eq. (55), $p_{0}(\omega)$ is a reference value that is taken to be the pressure by the point

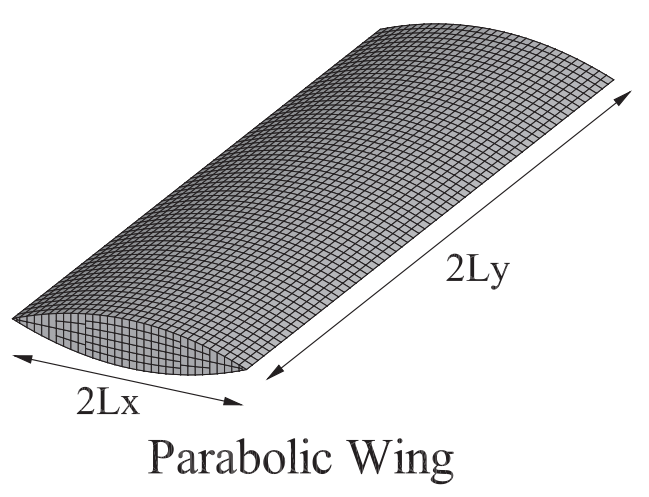

(a)

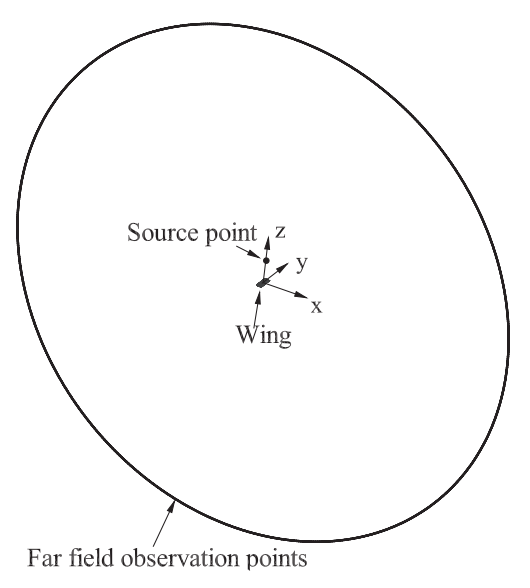

(b)
FIG. 3. A schematic of the computational setup. Left: dimensions of the parabolic wing and the surface mesh formed by 4364 quadrilateral elements, with $L_{x}=0.5, L_{y}=1.5$; Right: a diagram of the scattering body, source point, and the far field observation point which is on the $x-z$ plane and defined by $\hat{\boldsymbol{r}}=(\hat{R} \cos \theta, 0, \hat{R} \sin \theta)$ and $\hat{R}=105 L_{x}$. 
(a)

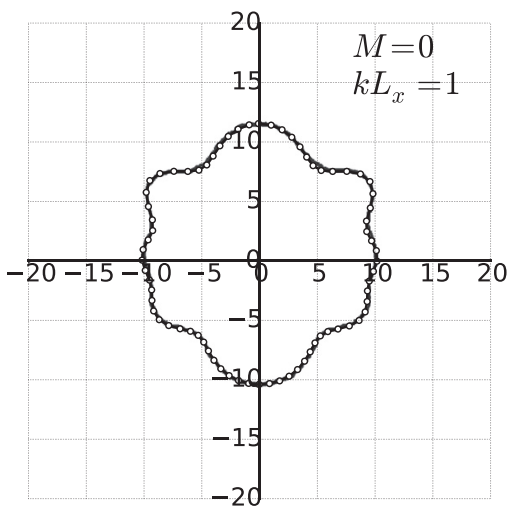

(c)

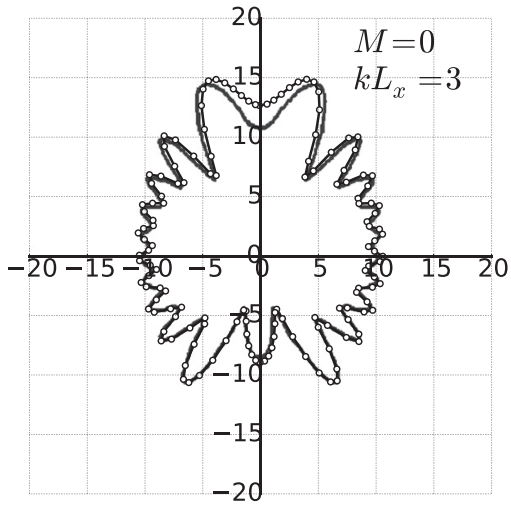

(e)

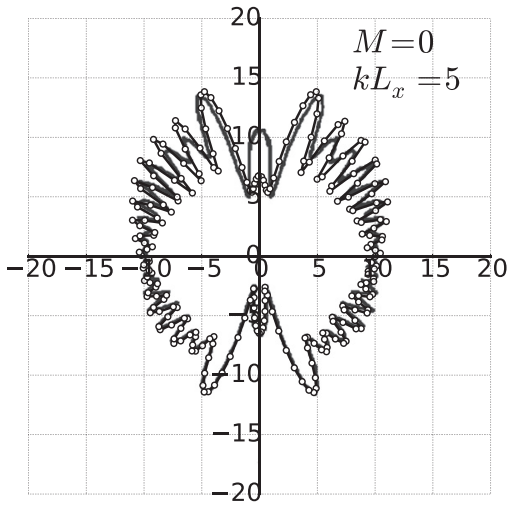

(b)

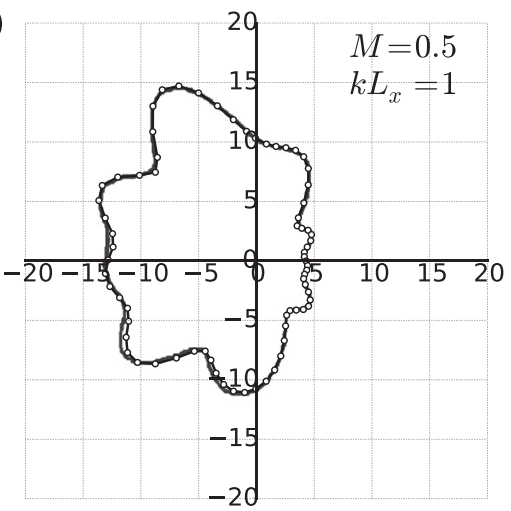

(d)

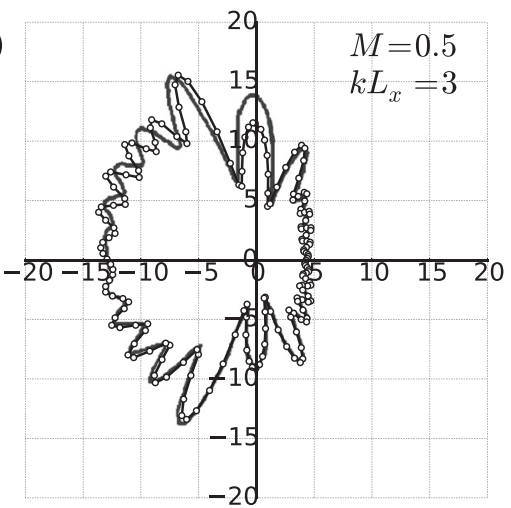

(f)

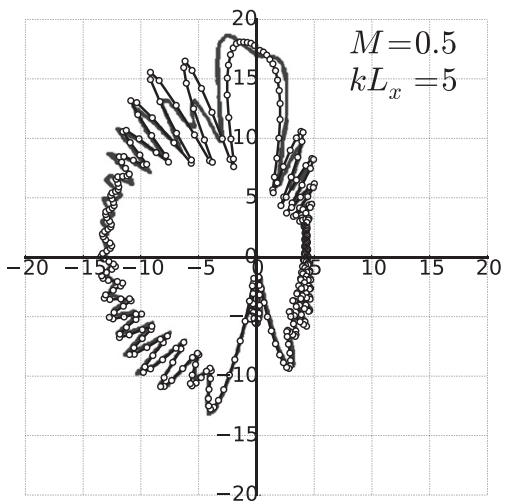

FIG. 4. Far field total pressure directivity patterns on the $x-z$ plane, for the frequencies and Mach numbers as indicated. The horizontal and vertical directions represent, respectively, the $x$ and $z$ directions as defined in Fig. 3. Lines with symbols: Current calculation; Solid lines: Results from Ref. 3. source (without the solid body) at the center point of the wing of coordinates $(0,0,0)$.

Figure 4 plots the directivity function $D(\theta)$ as polar graphs, in lines with symbols, at the three frequencies for the cases of Mach number $M=0$ and $M=0.5$. Effects of the mean flow on sound scattering are clearly seen. Also shown in Fig. 4 are the results from Ref. 3, in solid lines. We note that, at the low frequency $k L_{x}=1$, very good agreements are found for both the cases with and without flow. At higher frequencies, the two solutions in the downward direction (the shielded side below the scattering body) are also in very good agreements, while the results in the upward direction show some discrepancies. The discrepancies may be attributed to the fact that a much coarser mesh, only 46 elements and 120 nodal points, was used for the results in Ref. 3, as compared to 4364 elements used in the current computation. We also note that the results from Ref. 3 were computed using the usual normal velocity boundary condition (21). The fact that the results from both computations largely agree indicates that for the current example of a slender geometry, where normal component of the mean flow $M_{n}$ is small, the difference in the boundary condition does not have a large effect on the computational results. However, as pointed out earlier, the computation is much simplified by using the ZEF condition.

\section{CONCLUSIONS}

In this paper, we have considered the boundary condition to be used in time domain boundary integral equation 
analysis of acoustic scattering by solid bodies under a constant mean flow assumption. After an examination of the energy equation associated with the convective wave equation, it is proposed that an alternative boundary condition be defined by the requirement that the energy flux be zero at solid boundaries, instead of the usual boundary condition that the normal acoustic velocity component be zero. A new TDBIE is derived based on the proposed ZEF solid wall boundary condition. The new formulation differs from those found in the literature on the part of the boundary where the constant mean flow itself does not satisfy the solid surface boundary condition. In addition to conserving the acoustic energy, another significant advantage of the new equation is that it is considerably simpler than previous formulations. In particular, tangential derivatives of the solution on the solid surfaces are no longer required in the new formulation, which greatly simplifies numerical implementation and makes the separation of normal and tangential derivatives of the solution unnecessary. Moreover, to stabilize the TDBIE, a Burton-Miller reformulation is also derived. Numerical solutions and eigenvalue analysis are presented that demonstrate stability of the new formulation.

\section{ACKNOWLEDGMENTS}

F.Q.H. and M.E.P. are supported by a NASA Cooperative Agreement, NNX11AI63A. M.E.P. is also supported in part by an Old Dominion University Modeling and Simulation graduate fellowship. This work used the computational resources at the Old Dominion University ITS Turing cluster and the Extreme Science and Engineering Discovery Environment (XSEDE), which is supported by National Science Foundation Grant No. OCI-1053575. The authors would also like to thank the reviewers for constructive comments and suggestions.

\section{APPENDIX A: LIMIT OF WEAKLY-SINGULAR INTEGRAL}

By Eqs. (17) and (32), it is easy show that the modified normal derivatives $\left(\partial G_{0} / \partial \bar{n}\right)\left(\boldsymbol{r}_{s}, \boldsymbol{r}_{s}^{\prime}\right)$ and $\left(\partial^{2} G_{0} / \partial \bar{n}^{\prime} \partial \bar{n}\right)\left(\boldsymbol{r}_{s}, \boldsymbol{r}_{s}^{\prime}\right)$ have a singularity of order $O\left(1 /\left|\boldsymbol{r}_{s}-\boldsymbol{r}_{s}^{\prime}\right|\right)$ and $O\left(1 / \mid \boldsymbol{r}_{s}\right.$ $\left.\left.-\boldsymbol{r}_{s}^{\prime}\right)\left.\right|^{3}\right)$, respectively, which makes their surface integrals weakly singular and hyper-singular, respectively. In this appendix, we state some useful results.

For surface integrals involving $\partial G_{0} / \partial \bar{n}$, we have

$$
\frac{1}{4 \pi} \int_{S} \frac{\partial G_{0}}{\partial \bar{n}}\left(\boldsymbol{r}_{s}, \boldsymbol{r}^{\prime}\right) d \boldsymbol{r}_{S}= \begin{cases}0, & \boldsymbol{r}^{\prime} \in V, \text { exterior of } S \\ \frac{1}{2}, & \boldsymbol{r}^{\prime}=\boldsymbol{r}_{s}^{\prime} \in S \\ 1, & \boldsymbol{r}^{\prime} \in V^{-}, \text {interior of } S .\end{cases}
$$

The first and third equations in Eq. (A1) can be obtained by the fact that any constant can be a solution to the homogeneous convective wave equation with homogeneous normal derivative on the boundary for the interior domain $V^{-}$ enclosed by $S$. By substituting $\phi=1$ into Eq. (15) and noting the choice of the normal direction and the placement of $\boldsymbol{r}^{\prime}$, the first and third equation in Eq. (A1) follow immediately.
The second integral in Eq. (A1) becomes weakly singular when $\boldsymbol{r}^{\prime}$ approaches a point on surface $S$. This particular limit has been studied previous in the literature for a mean flow that is aligned with the $x$-coordinate. ${ }^{19,33}$ Here, we show the calculation for a general mean flow. Assuming $\boldsymbol{r}_{s}^{\prime}$ is a smooth point on $S$, consider modifying surface $S$ by a spherical surface of radius $\varepsilon$ and centered at $\boldsymbol{r}_{s}^{\prime}$ as shown in Fig. 5. The surface is assumed to be smooth at $\boldsymbol{r}_{s}^{\prime}$. If we denote the small hemispherical surface as $S_{\epsilon}$, we have

$$
\begin{aligned}
\lim _{\boldsymbol{r}^{\prime} \rightarrow \boldsymbol{r}_{s}^{\prime}} \int_{S} \frac{\partial G_{0}}{\partial \bar{n}}\left(\boldsymbol{r}_{s}, \boldsymbol{r}^{\prime}\right) d \boldsymbol{r}_{s}= & \lim _{\boldsymbol{r}^{\prime} \rightarrow \boldsymbol{r}_{s}^{\prime}} \int_{S-S_{\epsilon}} \frac{\partial G_{0}}{\partial \bar{n}}\left(\boldsymbol{r}_{s}, \boldsymbol{r}^{\prime}\right) d \boldsymbol{r}_{s} \\
& +\lim _{\boldsymbol{r}^{\prime} \rightarrow \boldsymbol{r}_{s}^{\prime}} \int_{S_{\epsilon}} \frac{\partial G_{0}}{\partial \bar{n}}\left(\boldsymbol{r}_{s}, \boldsymbol{r}^{\prime}\right) d \boldsymbol{r}_{s}
\end{aligned}
$$

Note that, for the surface integral on $S_{\epsilon}$, using Eq. (10), we have

$$
\begin{aligned}
\frac{\partial G_{0}}{\partial \bar{n}} & =-\alpha^{2} \frac{n_{1}\left(x_{s}-x_{s}^{\prime}\right)+n_{2}\left(y_{s}-y_{s}^{\prime}\right)+n_{3}\left(z_{s}-z_{s}^{\prime}\right)}{\bar{R}^{3}} \\
& =-\alpha^{2} \frac{\epsilon}{\bar{R}^{3}} .
\end{aligned}
$$

By the symmetry of $\bar{R}$ with respect to hemispheres $S_{\epsilon}$ and $S_{\epsilon}^{\prime}$, the complementary hemisphere of $S_{\epsilon}$, and by using a local spherical coordinate system which is centered at $\boldsymbol{r}_{s}^{\prime}$ and whose local $z$ direction coincides with mean flow $\boldsymbol{M}$, namely, $x_{s}-x_{s}^{\prime}=\epsilon \sin \nu \cos \theta, y_{s}-y_{s}^{\prime}=\epsilon \sin \nu \sin \theta, z_{s}-z_{s}^{\prime}=\epsilon \cos \nu$, we have

$$
\begin{aligned}
\lim _{\boldsymbol{r}^{\prime} \rightarrow \boldsymbol{r}_{s}^{\prime}} \int_{S_{\epsilon}} \frac{\partial G_{0}}{\partial \bar{n}} d \boldsymbol{r}_{s} & =-\alpha^{2} \int_{S_{\epsilon}} \frac{\epsilon}{\bar{R}^{3}} d \boldsymbol{r}_{s}=-\frac{\alpha^{2}}{2} \int_{S_{\epsilon}+S_{\epsilon}^{\prime}} \frac{\epsilon}{\bar{R}^{3}} d \boldsymbol{r}_{s} \\
& =-\frac{\alpha^{2}}{2} \int_{0}^{2 \pi} \int_{0}^{\pi} \frac{\epsilon^{3} \sin \nu}{\left(\epsilon^{2} \cos ^{2} \nu+\epsilon^{2} \alpha^{2} \sin ^{2} \nu\right)^{3 / 2}} d \nu d \theta \\
& =-\pi \alpha^{2} \int_{-1}^{1} \frac{1}{\left(\alpha^{2}+\left(1-\alpha^{2}\right) \chi^{2}\right)^{3 / 2}} d \chi=-2 \pi
\end{aligned}
$$

The last integral above can be found by direct integration. The second equation in Eq. (A1) follows as $\epsilon \rightarrow 0$ and by noting that, for $\boldsymbol{r}^{\prime} \in V$, the limit on the left hand side of Eq. (A2) is zero.

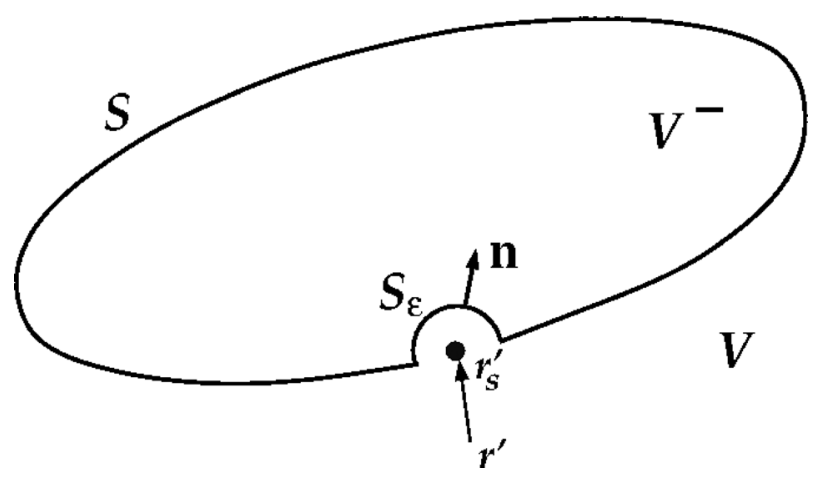

FIG. 5. A schematic diagram for a hemisphere that caps a surface point $\boldsymbol{r}_{s}^{\prime}$. Note that the normal vector is in the direction outward from the region of solution and into the body. 


\section{APPENDIX B: EVALUATION OF HYPER-SINGULAR INTEGRAL}

We consider the numerical evaluation of the regularized integral involving the double normal derivative of $G_{0}$ in Eq. (34) on a singular element $E_{i}$. Note that as $\boldsymbol{r}_{s} \rightarrow \boldsymbol{r}_{s}^{\prime}$, we have

$$
\begin{aligned}
\phi\left(\boldsymbol{r}_{s}, t_{R}^{\prime}\right)- & \phi\left(\boldsymbol{r}_{s}^{\prime}, t^{\prime}\right)+\frac{\bar{R}}{c \alpha^{2}} \frac{\partial \phi}{\partial t}\left(\boldsymbol{r}_{s}, t_{R}^{\prime}\right) \\
= & \nabla \phi\left(\boldsymbol{r}_{s}^{\prime}, t^{\prime}\right) \cdot\left(\boldsymbol{r}_{s}-\boldsymbol{r}_{s}^{\prime}\right)+\boldsymbol{\beta} \cdot\left(\boldsymbol{r}_{s}^{\prime}-\boldsymbol{r}_{s}\right) \\
& \times \frac{\partial \phi}{\partial t}\left(\boldsymbol{r}_{s}^{\prime}, t^{\prime}\right)+O\left(\left|\boldsymbol{r}_{s}-\boldsymbol{r}_{s}^{\prime}\right|^{2}\right) .
\end{aligned}
$$

Let the surface element $E_{i}$ be mapped to a local coordinate $(\xi, \eta) \in[-1,1] \times[-1,1]$, which is then in turn converted into a local polar coordinate $(r, \theta)$ centered at the collocation point $\boldsymbol{r}_{s}^{\prime}$. Denote the integrand for the integral in $(r, \theta)$ as

$$
\begin{aligned}
F(r, \theta)= & \left(\frac{\partial^{2} G_{0}}{\partial \bar{n}^{\prime} \partial \bar{n}}\right)\left(\phi\left(\boldsymbol{r}_{s}, t_{R}^{\prime}\right)-\phi\left(\boldsymbol{r}_{s}^{\prime}, t^{\prime}\right)+\frac{\bar{R}}{c \alpha^{2}} \frac{\partial \phi}{\partial t}\left(\boldsymbol{r}_{s}, t_{R}^{\prime}\right)\right) \\
& \times\left|\mathbf{r}_{\xi} \times \mathbf{r}_{\eta}\right| .
\end{aligned}
$$
the limit

By Eq. (B1), $F(r, \theta)$ is of order $O\left(1 / r^{2}\right)$ as $r \rightarrow 0$. Let

$$
\lim _{r \rightarrow 0} r^{2} F(r, \theta)=G(\theta) .
$$

It is easy to show that $\int_{0}^{2 \pi} G(\theta) d \theta=0$. Then we have the following for the integral on surface element $E_{i}$ :

$$
\begin{aligned}
\lim _{\epsilon \rightarrow 0} & \int_{0}^{2 \pi} \int_{\epsilon}^{r(\theta)} F(r, \theta) r d r d \theta \\
= & \lim _{\epsilon \rightarrow 0} \int_{0}^{2 \pi} \int_{\epsilon}^{r(\theta)}\left[\frac{r^{2} F(r, \theta)-G(\theta)}{r}+\frac{G(\theta)}{r}\right] d r d \theta \\
= & \int_{0}^{2 \pi} \int_{0}^{r(\theta)} \frac{r^{2} F(r, \theta)-G(\theta)}{r} d r d \theta \\
& +\lim _{\epsilon \rightarrow 0} \int_{0}^{2 \pi} G(\theta)[\ln r(\theta)-\ln \epsilon] d \theta \\
= & \int_{0}^{2 \pi} \int_{0}^{r(\theta)} \frac{r^{2} F(r, \theta)-G(\theta)}{r} d r d \theta+\int_{0}^{2 \pi} G(\theta) \ln r(\theta) d \theta .
\end{aligned}
$$

The final integrals above can now be evaluated using regular high-order numerical quadrature.

\section{APPENDIX C: EIGENVALUE BY MATRIX POWER ITERATION METHOD}

We describe a matrix power iteration method for finding the largest eigenvalue of Eq. (54). Let

$$
\boldsymbol{A}=\left[\begin{array}{cccccc}
-\boldsymbol{B}_{0}^{-1} \boldsymbol{B}_{1} & -\boldsymbol{B}_{0}^{-1} \boldsymbol{B}_{2} & \cdots & \cdots & -\boldsymbol{B}_{0}^{-1} \boldsymbol{B}_{J-1} & -\boldsymbol{B}_{0}^{-1} \boldsymbol{B}_{J} \\
\boldsymbol{I} & 0 & \cdots & \cdots & 0 & 0 \\
0 & \boldsymbol{I} & \cdots & \cdots & 0 & 0 \\
\cdots & \cdots & \cdots & \cdots & \cdots & \cdots \\
0 & 0 & \cdots & \cdots & 0 & 0 \\
0 & 0 & \cdots & \cdots & \boldsymbol{I} & 0
\end{array}\right] .
$$

Then, the power iteration method proceeds as follows. ${ }^{30}$

Given an arbitrary unit vector $\boldsymbol{e}^{(0)}$, and for $k=1,2, \ldots$, compute

$$
\begin{aligned}
& \boldsymbol{v}^{(k)}=\boldsymbol{A} \boldsymbol{e}^{(k-1)}, \\
& \boldsymbol{e}^{(k)}=\frac{\boldsymbol{v}^{(k)}}{\left\|\boldsymbol{v}^{(k)}\right\|_{2}},
\end{aligned}
$$

and eigenvalue

$$
\lambda^{(k)}=\left[\boldsymbol{e}^{(k)}\right]^{T} \boldsymbol{A} \boldsymbol{e}^{(k)}=\left[\boldsymbol{e}^{(k)}\right]^{T} \boldsymbol{v}^{(k+1)} .
$$

The iteration is stopped when $\left|\lambda^{(k)}-\lambda^{(k-1)}\right| /\left|\lambda^{(k)}\right|<\epsilon$, where $\epsilon$ is the tolerance and set to be $10^{-12}$. When the iteration is convergent, Eq. (C4) converges to the largest eigenvalue of $\boldsymbol{A}$.

Furthermore, if we denote

$$
\boldsymbol{e}^{(k)}=\left[\begin{array}{c}
\boldsymbol{e}_{J-1}^{(k)} \\
\boldsymbol{e}_{J-2}^{(k)} \\
\vdots \\
\vdots \\
\boldsymbol{e}_{1}^{(k)} \\
\boldsymbol{e}_{0}^{(k)}
\end{array}\right], \quad \boldsymbol{v}^{(k)}=\left[\begin{array}{c}
\boldsymbol{v}_{J-1}^{(k)} \\
\boldsymbol{v}_{J-2}^{(k)} \\
\vdots \\
\vdots \\
\boldsymbol{v}_{1}^{(k)} \\
\boldsymbol{v}_{0}^{(k)}
\end{array}\right]
$$

then, Eq. (C2) can also be computed through the following relations that save memory and storage:

$$
\begin{aligned}
\boldsymbol{v}_{J-1}^{(k)}= & -\boldsymbol{B}_{0}^{-1}\left[\boldsymbol{B}_{1} \boldsymbol{e}_{J-1}^{(k-1)}+\boldsymbol{B}_{2} \boldsymbol{e}_{J-2}^{(k-1)}+\cdots\right. \\
& \left.+\boldsymbol{B}_{J-1} \boldsymbol{e}_{1}^{(k-1)}+\boldsymbol{B}_{J} \boldsymbol{e}_{0}^{(k-1)}\right] \\
\boldsymbol{v}_{J-2}^{(k)}= & \boldsymbol{e}_{J-1}^{(k-1)}, \ldots, \boldsymbol{v}_{0}^{(k)}=\boldsymbol{e}_{1}^{(k-1)}
\end{aligned}
$$

We note that the iterative step shown in Eq. (C6) is the same as the MOT iteration Eq. (46) without the source term. Therefore, it can be carried out using the same computational scheme for Eq. (46).

${ }^{1} \mathrm{P}$. Zhang and T. W. Wu, "A hypersingular integral formulation for acoustic radiation in moving flows," J. Sound Vib. 206, 309-326 (1997).

${ }^{2}$ A. J. Burton and G. F. Miller, "The application of integral equation methods to the numerical solution of some exterior boundary-value problems," Proc. R. Soc. London, Ser. A 323, 201-210 (1971).

${ }^{3}$ M. K. Myers and J. S. Hausmann, "Computation of acoustic scattering from a moving rigid surface,” J. Acoust. Soc. Am. 91, 2594-2605 (1992).

${ }^{4} \mathrm{~F}$. Q. Hu, "An efficient solution of time domain boundary integral equations for acoustic scattering and its acceleration by Graphics Processing Units," AIAA paper 2013-2018 (2013).

${ }^{5}$ Y. W. Lee and D. J. Lee, "Derivation and implementation of the boundary integral formula for the convective acoustic wave equation in time domain,” J. Acoust. Soc. Am. 136, 2959-2967 (2014).

${ }^{6}$ F. Q. Hu, M. E. Pizzo, and D. M. Nark, "On the assessment of acoustic scattering and shielding by time domain boundary integral equation solutions," AIAA paper 2016-2779 (2016).

${ }^{7}$ A. A. Ergin, B. Shanker, and E. Michielssen, "Analysis of transient wave scattering from rigid bodies using a Burton-Miller approach," J. Acoust. Soc. Am. 106, 2396-2404 (1999).

${ }^{8}$ D. J. Chappell, P. J. Harris, D. Henwood, and R. Chakrabarti, "A stable boundary element method for modeling transient acoustic radiation," J. Acoust. Soc. Am. 120, 74-80 (2006). 
${ }^{9}$ B. P. Rynne, "Instabilities in time marching methods for scattering problems," Electromagnetics 6, 129-144 (1986).

${ }^{10}$ P. D. Smith, "Instabilities in time marching methods for scattering: Cause and rectification," Electromagnetics 10, 439-451 (1990).

${ }^{11}$ A. D. Jones and F. Q. Hu, "A three-dimensional time-domain boundary element method for the computation of exact Green's functions in acoustic analogy," AIAA paper 2007-3479 (2007).

${ }^{12} \mathrm{H}-\mathrm{W}$. Jiang and J-G. Ih, "Stabilization of time domain acoustic boundary element method for the exterior problem avoiding the nonuniqueness," J. Acoust. Soc. Am. 133, 1237-1244 (2013).

${ }^{13}$ H. A. Schenck, "Improved integral formulation for acoustic radiation problems," J. Acoust. Soc. Am. 44, 41-58 (1968).

${ }^{14} \mathrm{P}$. M. Morse and K. U. Ingard, Theoretical Acoustics (Princeton, University Press, Princeton, NJ, 1986).

${ }^{15}$ W. R. Morgans, "The Kirchhoff formula extended to a moving surface," Philos. Mag. 9, 141-161 (1930).

${ }^{16}$ J. E. Ffowcs Williams and D. L. Hawkings, "Sound generation by turbulence and surfaces in arbitrary motion," Philos. Trans. R. Soc. 264A, 321-342 (1969).

${ }^{17}$ Dowling, A. P. and J. E. Ffowcs Williams, Sound and Sources of Sound (Horwood Publishing, Westergaten, 1983).

${ }^{18}$ F. Farassat and M. K. Myers, "Extension of Kirchhoff formula to radiation from moving surfaces," J. Sound Vib. 123, 451-460 (1988).

${ }^{19}$ M. K. Myers and J. S. Hausmann, "On the application of the Kirchhoff formula for moving surfaces," J. Sound Vib. 139, 174-178 (1990).

${ }^{20}$ A. S. Lyrintzis, "Review: The use of Kirchhoff's method in computational aeroacoustics,” J. Fluids Eng. 116, 665-676 (1994).

${ }^{21}$ D. P. Lockard, "An efficient, two-dimensional implementation of the Ffowcs Williams and Hawkings equation,” J. Sound Vib. 229, 897-911 (2000)
${ }^{22}$ F. Q. Hu, Y. P. Guo, and A. D. Jones, "On the computation and application of exact Green's function in acoustic analogy," AIAA paper 20052986 (2005)

${ }^{23}$ Y. Guo, "Computation of sound propagation by boundary element method," NASA Contract Report, NAS1-00086-A003 (2005).

${ }^{24}$ A. Agarwal and P. J. Morris, "Prediction method for broadband noise from unsteady flow in a slat cove," AIAA J. 44, 301-312 (2006).

${ }^{25}$ M. H. Dunn and A. F. Tenetti, "Application of fast multipole methods to the NASA Fast Scattering code," AIAA paper 2008-2875 (2008).

${ }^{26}$ C. L. Morfey, "Acoustic energy in non-uniform flows," J. Sound Vib. 14, 159-179 (1971).

${ }^{27}$ M. K. Myers, "Transport of energy by disturbances in arbitrary flows," J. Fluid Mech. 226, 383-400 (1991).

${ }^{28} \mathrm{~W}$. Mohring, "Energy conservation, time reversal invariance and reciprocity in ducts with flow," J. Fluid Mech. 431, 223-237 (2001).

${ }^{29} \mathrm{~F}$. Q. Hu, "Further development of a time domain boundary integral equation method for aeroacoustic scattering computation," AIAA paper 20143194 (2014).

${ }^{30}$ G. H. Golub and C. F. Van Loan, Matrix Computation, 4th ed. (Johns Hopkins Studies in the Mathematical Sciences, Baltimore, 2013).

${ }^{31}$ S. J. Dodson, S. P. Walker, and M. J. Bluck, "Impicitness and stability of time domain integral equation scattering analysis," Appl. Comp. Eletromag. Soc. J. 13, 291-301 (1998).

${ }^{32}$ A. Iserles, A First Course in the Numerical Analysis of Differential Equations, 2nd ed. (Cambridge University Press, London, 2008).

${ }^{33}$ L. Long, "The compressible aerodynamics of rotating blades based on an acoustic formulation," NASA TP 2197 (1983). 